\title{
Density function for the second coefficient of the Hilbert-Kunz function on projective toric varieties
}

\author{
Mandira Mondal $^{1} \cdot$ Vijaylaxmi Trivedi $^{2}$
}

Received: 20 September 2018 / Accepted: 7 February 2019 / Published online: 23 March 2019

(c) Springer Science+Business Media, LLC, part of Springer Nature 2019

\section{Abstract}

We prove that, analogous to the Hilbert-Kunz density function, (used for studying the Hilbert-Kunz multiplicity, the leading coefficient of the Hibert-Kunz function), there exists a $\beta$-density function $g_{R, \mathbf{m}}:[0, \infty) \longrightarrow \mathbb{R}$, where $(R, \mathbf{m})$ is the homogeneous coordinate ring associated with the toric pair $(X, D)$, such that

$$
\int_{0}^{\infty} g_{R, \mathbf{m}}(x) \mathrm{d} x=\beta(R, \mathbf{m}),
$$

where $\beta(R, \mathbf{m})$ is the second coefficient of the Hilbert-Kunz function for $(R, \mathbf{m})$, as constructed by Huneke-McDermott-Monsky. Moreover, we prove, (1) the function $g_{R, \mathbf{m}}:[0, \infty) \longrightarrow \mathbb{R}$ is compactly supported and is continuous except at finitely many points, (2) the function $g_{R, \mathbf{m}}$ is multiplicative for the Segre products with the expression involving the first two coefficients of the Hilbert polynomials of the rings involved. Here we also prove and use a result (which is a refined version of a result by Henk-Linke) on the boundedness of the coefficients of rational Ehrhart quasipolynomials of convex rational polytopes.

Keywords Coefficients of Hilbert-Kunz function - Projective toric variety · Hilbert-Kunz density function · Rational Ehrhart quasi-polynomial · Rational Minkowski sum

Mathematics Subject Classification 13D40 $\cdot 13 \mathrm{H} 15 \cdot 14 \mathrm{M} 25 \cdot 52 \mathrm{~B} 20 \cdot 52 \mathrm{C} 22$

Mandira Mondal

mandiram@cmi.ac.in

Vijaylaxmi Trivedi

vija@math.tifr.res.in

1 Chennai Mathematical Institute, H1, SIPCOT IT Park, Siruseri, Kelambakkam 603103, India

2 School of Mathematics, Tata Institute of Fundamental Research, Homi Bhabha Road, Mumbai 400005, India 


\section{Introduction}

Let $R$ be a Noetherian ring of dimension $d$ and prime characteristic $p$, and let $I \subset R$ be an ideal such that $\ell(R / I)<\infty$. Let $M$ be a finitely generated $R$-module. Then the Hilbert-Kunz function of $M$ with respect to $I$ is defined by

$$
\operatorname{HK}(M, I)(n)=\ell\left(M / I^{[q]} M\right)
$$

where $q=p^{n}$, the ideal $I^{[q]}=n$th Frobenius power of $I$, and $\ell\left(M / I^{[q]} M\right)$ denotes the length of the $R$-module $M / I^{[q]} M$. The limit

$$
\lim _{n \rightarrow \infty} \frac{\ell\left(M / I^{\left[p^{n}\right]} M\right)}{q^{d}}
$$

exists (see [14]) and is called the Hilbert-Kunz multiplicity of $M$ with respect to the ideal $I$ (denoted by $e_{H K}(M, I)$ ). Thus $H K(M, I)(n)=e_{H K}(M, I) q^{d}+O\left(q^{d-1}\right)$. The Hilbert-Kunz multiplicity has been studied by many people since then.

In 2004, Huneke-McDermott-Monsky ([9]) proved the existence of a second coefficient for the Hilbert-Kunz function:

Theorem 1 (Theorem 1 of [9]) Let $R$ be an excellent normal Noetherian ring of dimension $d$ and characteristic $p$ and let $I \subset R$ be an ideal such that $\ell(R / I)<\infty$. Let $M$ be a finitely generated $R$-module. Then there exists a real number $\beta(M, I)$ such that

$$
\operatorname{HK}(M, I)(n)=e_{H K}(M, I) q^{d}+\beta(M, I) q^{d-1}+O\left(q^{d-2}\right) .
$$

They also found a relation with the divisor class group. This invariant was further studied by Kurano [10], and he proved there that $\beta(M, I)=0$ if $R$ is $\mathbb{Q}$-Gorenstein ring and $M$ is a Noetherian $R$-module of finite projective dimension. Later the above theorem of Huneke-McDermott-Monsky was generalised from normal rings to the rings satisfying $\left(R_{1}\right)$ condition by Chan-Kurano in [2] (also independently by Hochster-Yao in [8]). Later Bruns-Gubeladze in [1] have proved that HK function is a quasipolynomial and gave another proof of the existence of the constant second coefficient $\beta(R, \mathbf{m})$ for a normal affine monoid.

In order to study $e_{H K}(R, I)$, when $R$ is a standard graded $\operatorname{ring}(\operatorname{dim} R \geq 2)$ and $I$ is a homogeneous ideal of finite colength, the second author (in [18]) has defined the notion of Hilbert-Kunz density function, and obtained its relation with the HK multiplicity (stated in this paper as Theorem 5): The HK density function is a compactly supported continuous function $f_{R, I}:[0, \infty) \longrightarrow \mathbb{R}_{\geq 0}$ such that

$$
e_{H K}(R, I)=\int_{0}^{\infty} f_{R, I}(x) \mathrm{d} x .
$$

Moreover, there exists a sequence of functions $\left\{f_{n}(R, I):[0, \infty) \longrightarrow \mathbb{R}_{\geq 0}\right\}_{n}$ given by

$$
f_{n}(R, I)(x)=\frac{1}{q^{d-1}} \ell\left(R / I^{[q]}\right)\lfloor x q\rfloor
$$


such that $f_{n}$ converges uniformly to $f_{R, I}$.

The existence of a uniformly converging sequence makes the density function a more refined invariant (compared to $e_{H K}$ ) in the graded situation, and a useful tool, e.g., in suggesting a simpler approach to the HK multiplicity in characteristic 0 (see [20]), in studying the asymptotic growth of $e_{H K}\left(R, \mathbf{m}^{k}\right)$ as $k \rightarrow \infty$ (see [19]). Applying the theory of $\mathrm{HK}$ density functions to projective toric varieties (denoted here as toric pairs $(X, D)$ ), one obtains (Theorem 6.3 of [13]) an algebraic characterisation of the tiling property of the associated polytopes $P_{D}$ (in the ambient lattice) in terms of such asymptotic behaviour of $e_{H K}$.

In the light of Theorem 5 , one can speculate whether there exists a similar ' $\beta$-density function' $g_{R, \mathbf{m}}:[0, \infty) \rightarrow \mathbb{R}$ such that

$$
\int_{0}^{\infty} g_{R, \mathbf{m}}(x) \mathrm{d} x=\beta(R, I),
$$

which may similarly refine the $\beta$-invariant of [9] in the graded case.

We find that this is indeed true for a projectively normal toric pair $(X, D)$, i.e., $X$ a projectively normal toric variety over an algebraically closed field $K$ of characteristic $p>0$, with a very ample $T$-Cartier divisor $D$. Let $R$ be the homogeneous coordinate ring of $X$, with respect to the embedding given by the very ample line bundle $\mathcal{O}_{X}(D)$, and let $\mathbf{m}$ be the homogeneous maximal ideal of $R$. We also use the notation $\mathrm{rVol}_{d}$ to denote the $d$-dimensional relative volume function (see Definition 47).

We construct such a $\beta$-density function $g_{R, \mathbf{m}}$ as a limit of a 'uniformly' converging sequence of functions $\left\{g_{n}:[0, \infty) \longrightarrow \mathbb{R}\right\}_{n \in \mathbb{N}}$, which are given by

$$
g_{n}(\lambda)=\frac{1}{q^{d-2}}\left(\ell\left(R / \mathbf{m}^{[q]}\right)\lfloor\lambda q\rfloor-\tilde{f}_{n}(\lambda) q^{d-1}\right),
$$

where $\tilde{f}_{n}(\lambda)=f_{R, \mathbf{m}}(\lfloor\lambda q\rfloor / q)$ and $f_{R, \mathbf{m}}$ denotes the HK density function for $(R, \mathbf{m})$. From the construction, it follows that $g_{n}$ is a compactly supported function.

Recall that given a toric pair $(X, D)$, of dimension $d-1$, there is a convex lattice polytope $P_{D}$, a convex polyhedral cone $C_{D}$ and a bounded body $\mathcal{P}_{D}$ as in Notations 4 (such a bounded body was introduced by Eto (see [5]), in order to study the HK multiplicity for a toric ring, and he proved there that $e_{H K}$ is the relative volume of such a body). In [13], it was shown that the HK density function at $\lambda$ is the relative volume of the $\{z=\lambda\}$ slice of $\mathcal{P}_{D}$. Here we prove that $\beta$-density function at $\lambda$ is expressible in terms of the relative volume of the $\{z=\lambda\}$ slice of the boundary, $\partial\left(\mathcal{P}_{D}\right)$, of $\mathcal{P}_{D}$.

In this paper, the following is the main result.

Theorem 2 (Main Theorem) Let $(R, \mathbf{m})$ be the homogeneous coordinate ring of dimension $d \geq 3$, associated to the toric pair $(X, D)$. Then there exists a finite set $v\left(\mathcal{P}_{D}\right) \subseteq \mathbb{R}_{\geq 0}$ such that, for any compact set $V \subseteq \mathbb{R}_{\geq 0} \backslash v\left(\mathcal{P}_{D}\right)$, the sequence $\left\{\left.g_{n}\right|_{V}\right\}_{n}$ converges uniformly to $\left.g_{R, \mathbf{m}}\right|_{V}$, where $g_{R, \mathbf{m}}: \mathbb{R}_{\geq 0} \backslash v\left(\mathcal{P}_{D}\right) \longrightarrow \mathbb{R}$ is a continuous function given by

$$
g_{R, \mathbf{m}}(\lambda)=\operatorname{rVol}_{d-2}\left(\partial\left(\mathcal{P}_{D}\right) \cap \partial\left(C_{D}\right) \cap\{z=\lambda\}\right)-\frac{\operatorname{rVol}_{d-2}\left(\partial\left(\mathcal{P}_{D}\right) \cap\{z=\lambda\}\right)}{2} .
$$


Moreover, for $q=p^{n}$, we have

$$
\begin{aligned}
\int_{0}^{\infty} g_{n}(\lambda) \mathrm{d} \lambda & =\int_{0}^{\infty} g_{R, \mathbf{m}}(\lambda) \mathrm{d} \lambda+O\left(\frac{1}{q}\right) \text { and } \int_{0}^{\infty} \tilde{f}_{n}(\lambda) \mathrm{d} \lambda \\
& =\int_{0}^{\infty} f_{R, \mathbf{m}}(\lambda) \mathrm{d} \lambda+O\left(\frac{1}{q^{2}}\right)
\end{aligned}
$$

As a consequence, we get the following

Corollary 3 With the notations as above for a projectively normal toric pair $(X, D)$, we have

$$
\beta(R, \mathbf{m})=\int_{0}^{\infty} g_{R, \mathbf{m}}(\lambda) d \lambda=\operatorname{rVol}_{d-1}\left(\partial\left(\mathcal{P}_{D}\right) \cap \partial\left(C_{D}\right)\right)-\frac{\operatorname{rVol}_{d-1}\left(\partial\left(\mathcal{P}_{D}\right)\right)}{2}
$$

and the Hilbert-Kunz function of $R$ with respect to the maximal ideal $\mathbf{m}$ is given by

$$
\operatorname{HK}(R, \mathbf{m})(q)=e_{H K}(R, \mathbf{m}) q^{d}+\beta(R, \mathbf{m}) q^{d-1}+O\left(q^{d-2}\right) .
$$

Note that we can write

$$
g_{n}(\lambda)=\#\left(q \mathcal{P}_{D} \cap\{z=\lfloor\lambda q\rfloor\}\right) / q^{d-2}-(q) f_{R, \mathbf{m}}(\lfloor\lambda q\rfloor / q),
$$

where \# denotes the number of lattice points.

We show in Sect. 3 that $\overline{\mathcal{P}}_{D}=\left(\cup_{j} P_{j}\right) \backslash\left(\cup_{j, v} E_{j_{v}}\right)$, where $P_{j}$ and $E_{j_{v}}$ are certain rational convex polytopes with proper intersections. Then by applying the theory of Ehrhart quasi-polynomials and exhibiting that (in the case of a toric pair), the second coefficients of relevant Ehrhart quasi-polynomials are constant, we deduce that for $x \in$ $S=\left\{m / p^{n} \mid m, n \in \mathbb{N}\right\} \backslash v\left(\mathcal{P}_{D}\right)$, the sequence $\left\{g_{n}(x)\right\}_{n}$ is convergent and converges pointwise to $g_{R, \mathbf{m}}$. However, we still know neither the existence of $\lim _{n \rightarrow \infty} g_{n}(x)$, for every $x \in[0, \infty)$ (or for all $x$ except at finite number of points), nor that this limit is a continuous function. On the other hand, for $\lambda_{n}:=\lfloor\lambda q\rfloor / q \in S$, we have

$$
g_{n}(\lambda)=g_{R, \mathbf{m}}\left(\lambda_{n}\right)+\tilde{c}\left(\lambda_{n}\right) / q,
$$

where $\tilde{c}\left(\lambda_{n}\right)$ involves coefficients of Ehrhart quasi-polynomials of facets of $P_{j} \cap\{z=$ $\left.\lambda_{n}\right\}$. Therefore, to achieve a 'uniform convergence', we needed to prove the following:

Theorem 30 For a rational convex polytope $P$, where $P_{\lambda}:=P \cap\{z=\lambda\}$ and its Ehrhart quasi-polynomial is given by

$$
i\left(P_{\lambda}, q\right)=\sum_{i=0}^{\operatorname{dim}(P)} C_{i}\left(P_{\lambda}, q\right) q^{i}, \quad \text { if } \lambda q \in \mathbb{Z}_{\geq 0},
$$

there exist constant $\tilde{c}_{i}(P)$ such that every $\left|C_{i}\left(P_{\lambda}, q\right)\right| \leq \tilde{c}_{i}(P)$, for all $q \lambda \in \mathbb{Z}_{\geq 0}$ and $q=p^{n}$. 
We prove the result using the theory of lattice points in non-negative rational Minkowski sums. In fact we prove a general result about convex rational polytopes: Recall that, for rational convex polytopes $P_{1}, P_{2} \subset \mathbb{R}^{d}$ with $\operatorname{dim}\left(P_{1}+P_{2}\right)=d$, a well-known result of McMullen implies that the function $Q\left(P_{1}, P_{2} ;-\right): \mathbb{Q}_{\geq 0}^{2} \longrightarrow \mathbb{Z}$, given by $Q\left(P_{1}, P_{2}, ; \mathbf{r}\right)=\#\left(\left(r_{1} P_{1}+r_{2} P_{2}\right) \cap \mathbb{Z}^{d}\right)$ is a quasi-polynomial of degree $d$ (called the rational Ehrhart quasi-polynomial), i.e., we have

$$
Q\left(P_{1}, P_{2}, ; \mathbf{r}\right)=\sum_{\left(l_{1}, l_{2}\right) \in \mathbb{Z}_{\geq 0}^{2}, l_{1}+l_{2} \leq d} p_{l_{1}, l_{2}}(\mathbf{r}) r_{1}^{l_{1}} r_{2}^{l_{2}}
$$

such that (1) for some $\left(\tau_{1}, \tau_{2}\right) \in \mathbb{Q}_{>0}^{2}$ we have that $p_{l_{1}, l_{2}}\left(r_{1}, r_{2}\right)=p_{l_{1}, l_{2}}\left(r_{1}+\tau_{1}, r_{2}+\right.$ $\left.\tau_{2}\right)$, for every $\mathbf{r}=\left(r_{1}, r_{2}\right) \in \mathbb{Q}_{\geq 0}^{2}$ and (2) $p_{l_{1}, d-l_{1}}(\mathbf{r})$ is independent of $\mathbf{r}$.

Here we prove:

Theorem 28 There exists a decomposition $\left(0, \tau_{1}\right] \times\left(0, \tau_{2}\right]=\bigsqcup_{i=1}^{n} W_{i}$, where $W_{i}$ are locally closed subsets of $\mathbb{R}_{>0}^{2}$ and, for each $\left(l_{1}, l_{2}\right) \in \mathbb{Z}_{\geq 0}^{2}$, there exists a set of polynomials $\left.\left\{f_{l_{1}, l_{2}}^{i}: W_{i} \longrightarrow \mathbb{Q}\right\}\right\}_{i}$ such that

$$
p_{l_{1}, l_{2}}(\mathbf{r})=f_{l_{1}, l_{2}}^{i}(\mathbf{r}), \text { for every } \mathbf{r} \in W_{i} \cap \mathbb{Q}_{>0}^{2} .
$$

In particular, for all $\mathbf{r} \in \mathbb{Q}_{>0}^{2}$, there exist constants $C_{l_{1}, l_{2}}$ such that

$$
\left|p_{l_{1}, l_{2}}(\mathbf{r})\right| \leq C_{l_{1}, l_{2}} \text {, and } p_{l_{1}, d-l_{1}}(\mathbf{r})=C_{l_{1}, d-l_{1}} \text {. }
$$

The proof of Theorem 28 is a refinement of the proof of Theorem 1.3 of Henk-Linke ([7]), where they have proved (in our context) that the coefficients $p_{l_{1}, l_{2}}(-,-)$ are polynomials on the interior of the 2 -cells in $\mathbb{R}^{2}$, induced by the hyperplane arrangement (given by the support functions of $P_{1}$ and $P_{2}$ ). Since such cells do not cover $\mathbb{R}^{2}$ (or $\left.\left(0, \tau_{1}\right] \times\left(0, \tau_{2}\right]\right)$, and the complement contains line segments, the boundedness of the coefficients $p_{l_{1}, l_{2}}(-,-)$ cannot be directly obtained from the result of [7].

Note that their result was proved for Minkowski sums of any finite number of polytopes, which can also be easily refined using similar methods (see Remark 29). The similar result for rational Ehrhart quasi-polynomial for a single polytope was in an earlier paper of Linke (see Theorem 1.2, Corollary 1.5 and Theorem 1.6 of [11]).

Now the uniform convergence of the sequence $\left\{\left.g_{n}\right|_{V}\right\}_{n \in \mathbb{N}}$ to $\left.g\right|_{V}$, for any compact set $V$ of $[0, \infty) \backslash v\left(\mathcal{P}_{D}\right)$ is straightforward. Using the fact that the HK density function $f_{R, \mathbf{m}}$ is compactly supported, continuous, and (in this toric case) is a piecewise polynomial function, we deduce that

$$
\int_{0}^{\infty} \tilde{f}_{n}(\lambda) \mathrm{d} \lambda=\int_{0}^{\infty} f_{R, \mathbf{m}}(\lambda) \mathrm{d} \lambda+O\left(1 / q^{2}\right) .
$$

This and the similar approximation of the integral of the function $g_{R, \mathbf{m}}$ by the integrals of the functions $g_{n}$, as in the result stated above, implies that $\int g_{R, \mathbf{m}}(\lambda) \mathrm{d} \lambda$ is the second coefficient of the HK function of $(R, \mathbf{m})$. 
The paper is arranged as follows.

In Sect. 2, we recall notations and known results about projective toric varieties, including a brief review of the density function, treated in detail in [13].

In Sect. 3, we deal with the results about facets of the compact body $\overline{\mathcal{P}}_{D}$, for a toric pair $(X, D)$.

Section 4 is an independent section on rational convex polytopes. Here we study the coefficients of the (multivariate) rational Ehrhart quasi-polynomial and prove they take only finitely many polynomial values. Now, for a polytope $P$ and $P_{\lambda}=P \cap\{z=\lambda\}$, we relate the coefficients of the quasi-polynomial $i\left(P_{\lambda}, n\right)$, for all $\lambda \in \mathbb{R}_{\geq 0}$ such that $\lambda n \in$ $\mathbb{Z}$, with the coefficients of a fixed rational Ehrhart quasi-polynomial of Minkowski sum of two polytopes. In particular, we get a uniform bound on the coefficients of such $i\left(P_{\lambda}, n\right)$, which is important for the proof in Sect. 5 .

In Sect. 5 we present the main result about the $\beta$-density function.

In Sect. 6 we prove that the $\beta$-density function is a multiplicative function for Segre products of toric pairs. Here we also compute the $\beta$-density functions for $\left(\mathbb{P}^{2},-K\right)$, $\left(\mathbb{F}_{a}, c D_{1}+d D_{2}\right)$, where $-K$ is the anticanonical divisor on $\mathbb{P}^{2}$, and where $D_{1}$ and $D_{2}$ are a natural basis for the $T$-Cartier divisors of the Hirzebruch surface $\mathbb{F}_{a}$. In particular, one can compute the second coefficient of the Hilbert-Kunz function of the Segre products of $\left(\mathbb{P}^{2},-K\right),\left(\mathbb{F}_{a}, c D_{1}+d D_{2}\right)$ and other toric pairs of surfaces.

\section{Hilbert-Kunz density function on projective toric varieties}

Throughout the paper, we work over an algebraically closed field $K$ with char $p>0$ and follow the notations from [6]. Let $N$ be a lattice (which is isomorphic to $\mathbb{Z}^{n}$ ) and let $M=\operatorname{Hom}(N, \mathbb{Z})$ denote the dual lattice with a dual pairing $\langle$,$\rangle . Let T=\operatorname{Spec}(K[M])$ be the torus with character lattice $M$. Let $X(\Delta)$ be a complete toric variety over $K$ with fan $\Delta \subset N_{\mathbb{R}}$. We recall that the $T$-stable irreducible subvarieties of codimension 1 of $X(\Delta)$ correspond to one-dimensional cones (which are edges/rays of $\Delta$ ) of $\Delta$. If $\tau_{1}, \ldots, \tau_{n}$ denote the edges of the fan $\Delta$, then these divisors are the orbit closures $D_{i}=V\left(\tau_{i}\right)$. Let $v_{i}$ be the first lattice point along the edge $\tau_{i}$. A very ample $T$-Cartier divisor $D=\sum_{i} a_{i} D_{i}$ (note that $a_{i}$ are integers) determines a convex lattice polytope in $M_{\mathbb{R}}$ defined by

$$
P_{D}=\left\{u \in M_{\mathbb{R}} \mid\left\langle u, v_{i}\right\rangle \geq-a_{i} \text { for all } i\right\}
$$

and the induced embedding of $X(\Delta)$ in $\mathbb{P}^{r-1}$ is given by

$$
\phi=\phi_{D}: X(\Delta) \rightarrow \mathbb{P}^{r-1}, x \mapsto\left(\chi^{u_{1}}(x): \cdots: \chi^{u_{r}}(x)\right),
$$

where $P_{D} \cap M=\left\{u_{1}, u_{2}, \ldots, u_{r}\right\}$. For $(X(\Delta), D)$ and $P_{D}$ as above, consider the cone $\sigma$ in $N \times \mathbb{Z}$ whose dual $\sigma^{\vee}$ is the cone over $P_{D} \times 1$ in $M \times \mathbb{Z}$. Then the affine variety $U_{\sigma}$ corresponding to the cone $\sigma$ is the affine cone of $X(\Delta)$ in $\mathbb{A}_{K}^{r}$. Therefore, the homogeneous coordinate ring of $X(\Delta)$ (with respect to this embedding) is $K\left[\chi^{\left(u_{1}, 1\right)}, \ldots, \chi^{\left(u_{r}, 1\right)}\right]$. Note that there is an isomorphism of graded rings (see Proposition 1.1.9, [3]) 


$$
\frac{K\left[Y_{1}, \ldots, Y_{r}\right]}{I} \simeq K\left[\chi^{\left(u_{1}, 1\right)}, \ldots, \chi^{\left(u_{r}, 1\right)}\right]=: R,
$$

where the kernel $I$ is generated by the binomials of the form

$$
Y_{1}^{a_{1}} Y_{2}^{a_{2}} \cdots Y_{r}^{a_{r}}-Y_{1}^{b_{1}} Y_{2}^{b_{2}} \cdots Y_{r}^{b_{r}}
$$

where $a_{1}, \ldots, a_{r}, b_{1}, \ldots, b_{r}$ are non-negative integers satisfying the equations

$$
a_{1} u_{1}+\cdots+a_{r} u_{r}=b_{1} u_{1}+\cdots+b_{r} u_{r} \text { and } a_{1}+\cdots+a_{r}=b_{1}+\cdots+b_{r}
$$

Note that due to this isomorphism, we can consider $R=K[S]$ as a standard graded ring, where deg $\chi^{\left(u_{i}, 1\right)}=1$.

Throughout this section, we use the following

Notations 4 1. A toric pair $(X, D)$ means $X$ is a projective toric variety over a field $K$ of characteristic $p>0$, with a very ample $T$-Cartier divisor $D$. A toric pair is projectively normal if its coordinate ring (with respect to the embedding given by $D)$ is integrally closed domain.

2. The polytope $P_{D}$ or $P_{X, D}$ is the lattice polytope associated with the given toric $\operatorname{pair}(X, D)$ (as in (2)).

3. $f_{R, \mathbf{m}}=\operatorname{HKd}(R, \mathbf{m})$ is the HK density function of $R$ with respect to the ideal $\mathbf{m}$, where $R$ is the associated graded ring with the graded maximal ideal $\mathbf{m}$ (as in (3)).

4. Let $C_{D}$ denote the convex rational polyhedral cone spanned by $P_{D} \times 1$ in $M_{\mathbb{R}} \times \mathbb{R}$.

5. Let

$$
\mathcal{P}_{\mathcal{D}}=\left\{p \in C_{D} \mid p \notin(u, 1)+C_{D} \text {, for every } u \in P_{D} \cap M\right\}
$$

6. For a set $A \subset M_{\mathbb{R}} \simeq \mathbb{R}^{d}$, we denote

$$
A \cap\{z=\lambda\}:=A \cap\left\{(\mathbf{x}, \lambda) \mid \mathbf{x} \in \mathbb{R}^{d-1}\right\} .
$$

Theorem 5 (Theorem 1.1 in [18]) Let $R$ be a standard graded Noetherian ring of dimension $d \geq 2$ over an algebraically closed field $K$ of characteristic $p>0$, and let $I \subset R$ be a homogeneous ideal such that $\ell(R / I)<\infty$. For $n \in \mathbb{N}$ and $q=p^{n}$, let $f_{n}(R, I):[0, \infty) \longrightarrow[0, \infty)$ be defined as

$$
f_{n}(R, I)(x)=\frac{1}{q^{d-1}} \ell\left(R / I^{[q]}\right)\lfloor x q\rfloor
$$

(here $\ell\left(R / I^{[q]}\right)_{\lfloor x q\rfloor}$ denote the dimension of the $K$-vector space $\left.\left(R / I^{[q]}\right)_{\lfloor x q\rfloor}\right)$. Then $\left\{f_{n}(R, I)\right\}_{n}$ converges uniformly to a compactly supported continuous function $f_{R, I}:[0, \infty) \longrightarrow[0, \infty)$, where $f_{R, I}(x)=\lim _{n \rightarrow \infty} f_{n}(R, I)(x)$ and

$$
e_{H K}(R, I)=\int_{0}^{\infty} f_{R, I}(x) \mathrm{d} x
$$


We recall Theorem (1.1) from [13]:

Theorem 6 Let $(X, D)$ be a toric pair with associated ring $(R, \mathbf{m})$, and $P_{D}, C_{D}, \mathcal{P}_{D}$ as in Notations 4. Then the Hilbert-Kunz density function of $(R, \mathbf{m})$ is given by the sectional volume of $\overline{\mathcal{P}}_{D}$, i.e.

$$
f_{R, \mathbf{m}}(\lambda)=\operatorname{rVol}_{d-1}\left(\overline{\mathcal{P}}_{D} \cap\{z=\lambda\}\right), \text { for } \lambda \in[0, \infty) .
$$

Moreover, $f_{R, \mathbf{m}}$ is given by a piecewise polynomial function.

\section{The boundary of $\mathcal{P}_{D}$}

Recall that (Notations 4) associated with a given toric pair $(X, D)$, we have a convex polytope $P_{D}$, a convex polyherdral cone $C_{D}$ and a bounded set $\mathcal{P}_{D} \subset \mathbb{R}^{d}$. In [13], we had written a decomposition $C_{D}=\cup_{j} F_{j}$, where $F_{j}^{\prime} s$ are $d$-dimensional cones such that, each $P_{j}:=F_{j} \cap \overline{\mathcal{P}}_{D}$ is a convex rational polytope and is a closure of $P_{j}^{\prime}:=F_{j} \cap \mathcal{P}_{D}$. To study the boundary of $\mathcal{P}_{D}$ we need a set of lemmas about the facets of $P_{j}^{\prime} s$. We also assume without loss of generality that $d \geq 3$, as $d=2$ corresponds to $\left(\mathbb{P}^{1}, \mathcal{O}_{\mathbb{P}^{1}}(n)\right)$, for $n \geq 1$, which is easy to handle directly.

Notations 7 1. $L\left(P_{D}\right)=P_{D} \cap M=$ the (finite) set of lattice points of $P_{D}$.

2. For a convex polytope $Q$, let $v(Q)=$ vertices of $Q$ \} and $F(Q)=\{$ facets of $Q$ \}.

3. $A(F)=$ the affine hull of $F$ in $\mathbb{R}^{d}$, for a set $F \subseteq \mathbb{R}^{d}$. Recall that affine hull of a set $S \subseteq \mathbb{R}^{n}$ is the smallest affine set containing $S$, i.e. $A(S)=\left\{\sum_{i=1}^{m} a_{i} s_{i} \mid m \in\right.$ $\left.\mathbb{N}, s_{i} \in S, a_{i} \in \mathbb{R}, \sum_{i=1}^{m} a_{i}=1\right\}$.

4. For a polytope $F, \operatorname{dim} F:=\operatorname{dim} A(F)$.

5. $F_{j} \in\{d$-dimensional cones $\}$, which is the closure of a connected component of $C_{D} \backslash \cup_{i u} H_{i u}$, where the hyperplanes $H_{i u}$ are given by

$$
H_{i u}=\text { the affine hull of }\left\{\left(v_{i k}, 1\right),(u, 1),(\mathbf{0}) \mid v_{i k} \in v\left(C_{0 i}\right), \quad u \in P_{D} \cap M\right\},
$$

where $C_{0 i} \in\left\{(d-3)\right.$ dimensional faces of $\left.P_{D}\right\}$ and $\mathbf{0}$ is the origin of $\mathbb{R}^{d}$.

6. For a subset $A \subseteq C_{D}$, we denote

(a) $\partial_{C} A=$ boundary of $A$ in the subspace topology of $C_{D}$ (thinking of $C_{D} \subset \mathbb{R}^{d}$ ) and

(b) $\partial(A)=$ the boundary of $A$ in $\mathbb{R}^{d}$.

In particular $\partial_{C} A \subseteq \partial(A)$.

7. For $u \in L\left(P_{D}\right)$, let $C_{u}=(u, 1)+C_{D}$ and let

$$
P_{j}^{\prime}=F_{j} \cap \cap_{u \in L\left(P_{D}\right)}\left((u, 1)+C_{D}\right)^{c}=F_{j} \cap \cap_{u \in L\left(P_{D}\right)}\left[C_{D} \backslash C_{u}\right]
$$

which is a convex set (Lemma 4.5 of [13]).

8. Let $P_{j}=\overline{F_{j} \cap \cap_{u \in L\left(P_{D}\right)}\left(C_{D} \backslash C_{u}\right)}$ the convex rational polytope which is the closure of $P_{j}^{\prime}$ in $C_{D}$ (which equals the closure in $\mathbb{R}^{d}$ ). 
Therefore,

$$
\mathcal{P}_{D}=\cup_{j=1}^{s} P_{j}^{\prime} \text { and } \overline{\mathcal{P}}_{D}=\cup_{j=1}^{s} P_{j},
$$

where $P_{1}, \ldots, P_{s}$ are distinct polytopes, whose interiors are disjoint.

Note that

$$
P_{j}=\overline{F_{j} \backslash \cup_{u \in L\left(P_{D}\right)} C_{u}}=\overline{\bigcap_{u \in L\left(P_{D}\right)} F_{j} \backslash C_{u}} .
$$

Lemma 8 For each $P_{j}$ as in Notations 7, we have

1. $P_{j}=P_{j}^{\prime} \sqcup\left(\cup_{u \in L\left(P_{D}\right)}\left\{\partial_{C}\left(C_{u}\right) \cap P_{j}\right\}\right)$.

2. For any $u \in L\left(P_{D}\right)$, we have $\partial_{C}\left(C_{u}\right) \cap P_{j}=\cup_{\left\{F^{\prime} \mid F^{\prime} \in F\left(C_{u}\right), F^{\prime} \nsubseteq \partial\left(C_{D}\right)\right\}} F^{\prime} \cap P_{j}$.

Proof (1) We only need to prove that $P_{j} \subseteq P_{j}^{\prime} \bigsqcup_{u}\left\{\partial_{C}\left(C_{u}\right) \cap P_{j}\right\}$, as the other way inclusion is obvious.

Let us denote $\mathcal{P}_{D}=\cap_{u \in P_{D} \cap \mathbb{Z}^{d-1}}\left[C_{D} \backslash C_{u}\right]$ by $U$. Then $P_{j}^{\prime}=F_{j} \cap U$ and $P_{j}=$ $\overline{F_{j} \cap U}$. It is easy to check that $\overline{F_{j} \cap U} \subseteq\left(F_{j} \cap U\right) \cup\left(\partial_{C} U \cap \overline{F_{j} \cap U}\right)$. Now

$$
\partial_{C} U \subseteq \cup_{u} \partial_{C}\left(C_{u}\right) \Longrightarrow\left(\partial_{C} U\right) \cap \overline{F_{j} \cap U} \subseteq \cup_{u \in L\left(P_{D}\right)}\left\{\partial_{C}\left(C_{u}\right) \cap P_{j}\right\}
$$

This proves Assertion (1).

(2) We leave this to the reader. This proves lemma.

Lemma 9 For any facet $F \in F\left(P_{j}\right)$, we have one and only one of the following possibilities:

1. $F \subset F_{j_{i}}$, for some facet $F_{j_{i}} \in F\left(F_{j}\right)$ : (i) In this case $F=A\left(F_{j_{i}}\right) \cap P_{j}=F_{j_{i}} \cap P_{j}$ and

(ii) $\operatorname{dim}\left[A(F) \cap\left(\cup_{u \in L\left(P_{D}\right)} \partial_{C}\left(C_{u}\right) \cap P_{j}\right)\right] \leq d-2$.

2. $F \subset F_{u_{v}}$, for some facet $F_{u_{v}} \in F\left(C_{u}\right)$ and $u \in L\left(P_{D}\right)$. In this case $F=$ $P_{j} \cap F_{u_{v}}=P_{j} \cap A\left(F_{u_{v}}\right)$, where $F_{u_{v}} \nsubseteq \partial\left(C_{D}\right)$.

Proof Note $P_{j}^{\prime}=F_{j} \cap\left(\cap_{u \in L\left(P_{D}\right)}\left[C_{D} \backslash C_{u}\right]\right)$. Therefore,

$F \subset \partial\left(P_{j}\right) \subseteq \partial\left(F_{j}\right) \cup \cup_{u \in L\left(P_{D}\right)} \partial\left[C_{u}\right]=\cup_{F_{j_{i}} \in F\left(F_{j}\right)} F_{j_{i}} \cup \cup_{u \in L\left(P_{D}\right)} \cup_{F_{u_{v}} \in F\left(C_{u}\right)} F_{u_{v}}$.

This implies $d-1=\operatorname{dim} F=\max \left\{\operatorname{dim}\left(F_{j_{i}} \cap F\right), \operatorname{dim}\left(F_{u_{v}} \cap F\right)\right\}_{j_{i}, u_{v}}$. Hence at least one of the sets, $F_{j_{i}} \cap F$ or $F_{u_{v}} \cap F$, for some $j_{i}$ or $u_{v}$, is of dimension $d-1$. This implies either $A(F)=A\left(F_{j_{i}}\right)$ or $A(F)=A\left(F_{u_{v}}\right)$.

(1) Let $F \in F\left(P_{j}\right)$ such that $F \subseteq A\left(F_{j_{i}}\right)$, for some $j_{i}$. Then $F=P_{j} \cap A\left(F_{j_{i}}\right)=$ $P_{j} \cap F_{j} \cap A\left(F_{j_{i}}\right)=P_{j} \cap F_{j_{i}}$. In particular $F \subseteq F_{j_{i}}$. This proves Assertion (1) (i).

Suppose given $F \in F\left(P_{j}\right)$ such that $F \subseteq A\left(F_{j_{i}}\right)$ and for some $u \in L\left(P_{D}\right)$, $\operatorname{dim}\left[A(F) \cap\left(\partial_{C}\left(C_{u}\right) \cap P_{j}\right)\right]=d-1$. Then $\operatorname{dim}\left(A(F) \cap F_{u_{v}} \cap P_{j}\right)=d-1$, 
for some $F_{u_{v}} \in F\left(C_{u}\right)$ such that $F_{u_{v}} \nsubseteq \partial\left(C_{D}\right)$. Then $A(F)=A\left(F_{u_{v}}\right)=A\left(F_{j_{i}}\right)$, where $A\left(F_{j_{i}}\right)$ is a hyperplane passing through the origin $\mathbf{0}$ of $\mathbb{R}^{d}$, and therefore, $A(F)$ is a vector subspace of $\mathbb{R}^{d}$. Also $F_{u_{v}}=(u, 1)+F^{\prime}$, for some $F^{\prime} \in F\left(C_{D}\right)$. Therefore, $A(F)=(u, 1)+A\left(F^{\prime}\right)$. Hence $(u, 1)+\mathbf{y}=\mathbf{0}$, for some $\mathbf{y} \in A\left(F^{\prime}\right)$, which implies $A(F)=A\left(F^{\prime}\right)$. Therefore, $F_{u_{v}} \subseteq A(F) \cap C_{D}=F^{\prime} \subseteq \partial\left(C_{D}\right)$, which is a contradiction. This implies (1) (ii) and hence the first assertion.

(2) We first prove the following

Claim For any $F_{j}$ and for a facet $F^{\prime \prime} \in F\left(C_{u}\right)$, where $u \in L\left(P_{D}\right)$, if $F^{\prime \prime} \cap F_{j}^{o} \neq \phi$, then $F^{\prime \prime} \cap F_{j}=A\left(F^{\prime \prime}\right) \cap F_{j}$, where $F_{j}^{o}=F_{j} \backslash \partial\left(F_{j}\right)$.

Proof of the Claim Recall (see Lemma 4.5 of [13]) that $F_{j} \backslash C_{u}$ is a convex set, for any $u \in L\left(P_{D}\right)$.

If $\left(A\left(F^{\prime \prime}\right) \cap F_{j}\right) \backslash F^{\prime \prime} \cap F_{j} \neq \phi$, then there exists $x \in\left(\partial F^{\prime \prime}\right) \cap F_{j}^{o}$ and an open set (in $\left.\mathbb{R}^{d}\right) B_{x} \subseteq F_{j}^{o}$ such that $B_{x} \cap C_{u} \cap F_{j}^{o} \neq \phi$. Hence there is another facet $F^{\prime} \in F\left(C_{u}\right)$ such that $F^{\prime} \cap F_{j}^{o} \neq \phi\left(x \in \partial\left(F^{\prime}\right) \cap F_{j}^{o}\right)$.

Note that $\operatorname{dim}\left(F^{\prime} \cap F_{j}^{o}\right)=\operatorname{dim}\left(F^{\prime \prime} \cap F_{j}^{o}\right)=d-1$ and $\operatorname{dim}\left(F^{\prime} \cap F^{\prime \prime}\right) \leq d-2$. Hence we choose $x_{1} \in F^{\prime \prime} \cap F_{j}^{o}$ and $x_{2} \in F^{\prime} \cap F_{j}^{o}$ such that $x_{1} \neq x_{2}$. Then $t x_{1}+(1-t) x_{2} \subseteq$ $F_{j} \cap C_{u}$, for $0 \leq t \leq 1$. Now we can also choose small enough neighourhoods (open in $\mathbb{R}^{d}$ ) $B_{x_{1}}$ and $B_{x_{2}}$ of $x_{1}$ and $x_{2}$, respectively, which are contained in $F_{j}$. Let $L$ be the affine line through $x_{1}$ and $x_{2}$. Now, the line segment of $L$ with end points $x_{1}^{\prime} \in B_{x_{1}} \cap L \cap C_{u}^{c}$ and $x_{2}^{\prime} \in B_{x_{2}} \cap L \cap C_{u}^{c}$, passes through $C_{u}$, which contradicts the convexity property of $F_{j} \backslash C_{u}$. Hence the claim.

Suppose $F \in F\left(P_{j}\right)$ such that $F \nsubseteq A\left(F_{j_{i}}\right)$, for any $F_{j_{i}} \in F\left(F_{j}\right)$. Then there exists $F_{u_{v}} \in F\left(C_{u}\right)$, for some $u \in L\left(P_{D}\right)$, such that $\operatorname{dim}\left(F \cap F_{u_{v}}\right)=d-1$. This implies $A(F)=A\left(F_{u_{v}}\right)$, and therefore, $F_{u_{v}} \nsubseteq A\left(F_{j_{i}}\right)$, for any $F_{j_{i}}$. On the other hand $F \cap F_{u_{v}} \subseteq F_{j}$. Hence $F_{u_{v}} \cap F_{j}^{o} \neq \phi$, which implies, by the above claim that $A\left(F_{u_{v}}\right) \cap F_{j}=F_{u_{v}} \cap F_{j}$. Therefore,

$$
F \subseteq A\left(F_{u_{v}}\right) \cap P_{j} \cap F_{j}=F_{u_{v}} \cap F_{j} \cap P_{j}=F_{u_{v}} \cap P_{j} \subseteq F
$$

Moreover, by definition $F \cap F_{u_{v}} \subset P_{j} \backslash P_{j}^{\prime}$, and therefore, by Lemma $8, F_{u_{v}} \nsubseteq$ $\partial\left(C_{D}\right)$. This proves the second assertion and hence the lemma.

Lemma 10 If $P_{i} \neq P_{j}$, then $\operatorname{dim}\left(P_{i} \cap P_{j}\right) \leq d-1$. Moreover, if $\operatorname{dim}\left(P_{i} \cap P_{j}\right)=d-1$, then $P_{i} \cap P_{j} \in F\left(P_{i}\right) \cap F\left(P_{j}\right)$, that is, $P_{i}$ and $P_{j}$ meet along a common facet.

Proof We know $P_{i} \cap P_{j} \subseteq F_{i} \cap F_{j}$, where $\operatorname{dim}\left(F_{i} \cap F_{j}\right) \leq d-1$. If $\operatorname{dim}\left(P_{i} \cap P_{j}\right)=$ $d-1$, then $F_{i} \cap F_{j} \in F\left(F_{i}\right) \cap F\left(F_{j}\right)$. Let $F=F_{i} \cap F_{j}$; then $F_{i} \cap A(F)=F_{j} \cap A(F)=$ $F$. But $P_{i}=\overline{F_{i} \cap U}$ and $P_{j}=\overline{F_{j} \cap U}$. Therefore, $P_{i} \cap A(F)=\overline{F_{i} \cap U} \cap A(F)=$ $\overline{F_{i} \cap U \cap A(F)}=\overline{F \cap U}=P_{j} \cap A(F)$. This proves the lemma.

\section{Lemma 11}

(1) $\partial\left(\mathcal{P}_{D}\right)=\cup_{\left\{F \in F\left(P_{j}\right) \mid F \neq P_{i} \cap P_{j}\right\}} F$. In particular

(2) $\partial\left(\mathcal{P}_{D}\right)=\bigcup_{\left\{F \in F\left(C_{D}\right)\right\}} F \cap \overline{\mathcal{P}}_{D} \cup \bigcup_{\left\{F \in F\left(C_{u}\right), u \in L\left(P_{D}\right)\right\}} F \cap \overline{\mathcal{P}}_{D}$. 
Proof Note $\partial\left(\mathcal{P}_{D}\right)=\partial\left(\overline{\mathcal{P}}_{D}\right) \subseteq \cup_{j} \cup_{F \in F\left(P_{j}\right)} F$. Moreover, $\cup_{j, \nu} E_{j v} \subseteq \partial\left(\mathcal{P}_{D}\right)$, where

$$
\left\{E_{j v}\right\}=\left\{F \in F\left(P_{j}\right) \mid F \subseteq F^{\prime}, F^{\prime} \in F\left(C_{u}\right), u \in L\left(P_{D}\right)\right\} \subseteq \overline{\mathcal{P}}_{D} \cap \overline{\mathcal{P}_{D}^{c}} \subseteq \partial\left(\mathcal{P}_{D}\right) .
$$

Let $V=\cup_{F}\left\{F \mid F \in F\left(P_{j}\right), \quad F \neq P_{i} \cap P_{j}\right.$, for any $\left.j\right\}$. Then $\cup_{j, v} E_{j v} \subseteq V$ (see Lemma 9). Therefore, $\partial\left(\mathcal{P}_{D}\right) \backslash V \subseteq \cup_{F}\left\{F \mid F=P_{i} \cap P_{j}\right\}$. If $\partial\left(\mathcal{P}_{D}\right) \backslash V \neq \phi$, then there exists $(d-1)$-dimensional open ball $B_{d-1} \subseteq \partial \mathcal{P}_{D} \cap P_{i} \cap P_{j}$, for some $P_{i} \neq P_{j}$. Therefore, $P_{i} \backslash V$ and $P_{j} \backslash V$ are nonempty open sets of $P_{i}$ and $P_{j}$, respectively. Hence, we can choose an open set $B_{d}$ such that

$$
B_{d}=\left[B_{d} \cap\left(P_{i} \backslash V\right)\right] \cup\left[B_{d} \cap\left(P_{j} \backslash V\right)\right] \cup\left[B_{d} \cap\left(P_{i} \cap P_{j}\right)\right],
$$

where $B_{d} \cap P_{i} \cap P_{j} \subseteq B_{d-1}$. Since $B_{d-1} \subseteq P_{i} \cap P_{j}$, we have $B_{d-1} \cap \overline{\mathcal{P}}_{D}{ }^{c}=\phi$, which implies $B_{d-1} \cap \partial\left(\mathcal{P}_{D}\right)=\phi$, hence a contradiction. Therefore, $\partial\left(\mathcal{P}_{D}\right)=V$. This proves Assertion (1). Now

$$
\begin{aligned}
\partial\left(\mathcal{P}_{D}\right) & =\bigcup_{\left\{F \in F\left(P_{j}\right), F \subseteq A\left(F_{j}\right), F \neq P_{i} \cap P_{j}\right\}} F \cup \bigcup_{j, v}\left\{E_{j v}\right\} \\
& =\bigcup_{\left\{F \in F\left(C_{D}\right)\right\}} F \cap \overline{\mathcal{P}}_{D} \cup \bigcup_{\left\{F \in F\left(C_{u}\right), u \in L\left(P_{D}\right)\right\}} F \cap \overline{\mathcal{P}}_{D} .
\end{aligned}
$$

This proves the lemma.

Notations 12 In the rest of the paper, for a bounded set $Q \subset \mathbb{R}^{d}$ and for $n, m \in \mathbb{N}$, we define

$$
i(Q, n, m):=\#\left(n Q \cap\{z=m\} \cap \mathbb{Z}^{d}\right),
$$

where $z$ is the $d^{\text {th }}$ coordinate function on $\mathbb{R}^{d}$.

Remark 13 From Lemma 8, it follows that

$$
P_{j}=P_{j}^{\prime} \bigsqcup\left(\cup_{\gamma} E_{j \gamma}\right) \text {, where }\left\{E_{j \gamma}\right\}=\left\{F \in F\left(P_{j}\right)\right\}_{\left\{F \subseteq F^{\prime}, F^{\prime} \in F\left(C_{u}\right), u \in L\left(P_{D}\right)\right\}}
$$

and $E_{j v} \nsubseteq \partial\left(C_{D}\right)$. Note, $E_{j \gamma} \cap P_{i}^{\prime}=\phi$, for every $i$, as $P_{i}^{\prime} \subseteq \cup_{u \in L\left(P_{D}\right)} C_{D} \backslash C_{u}$. In particular,

$$
i\left(\mathcal{P}_{D}, n, m\right)=i\left(\cup_{j} P_{j}^{\prime}, n, m\right)=i\left(\cup_{j} P_{j}, n, m\right)-i\left(\cup_{j \gamma} E_{j \gamma}, n, m\right) .
$$

Therefore, we have

$$
\begin{aligned}
i\left(\mathcal{P}_{D}, n, m\right)= & \sum_{j} i\left(P_{j}, n, m\right)-\sum_{j<k} i\left(P_{j} \cap P_{k}, n, m\right) \\
& -\sum_{j, \gamma} i\left(E_{j \gamma}, n, m\right)+\sum_{\alpha \in I_{1}} \epsilon_{\alpha} i\left(Q_{\alpha}^{\prime}, n, m\right),
\end{aligned}
$$


where $\left\{Q_{\alpha}^{\prime}\right\}_{\alpha \in I_{1}}$ runs over a certain finite set of polytopes of dimension $\leq d-1$ : either $Q_{\alpha}^{\prime}=P_{j_{1}} \cap \cdots \cap P_{j_{l}}$, for distinct $P_{j_{i}}^{\prime} s$, where $l \geq 3$, or $Q_{\alpha}^{\prime}=E_{j_{1} \gamma_{1}} \cap \cdots \cap E_{j_{l} \gamma_{l}}$, for distinct $E_{j_{i} \gamma_{i}}^{\prime} s$, and $l \geq 2$. Note that $\epsilon_{\alpha} \in\{1,-1\}$, depending on $\alpha \in I_{1}$.

Lemma 14 Let $Q$ be a convex polytope such that $Q \subseteq F$, for some facet $F \in F\left(P_{j}\right)$, where $1 \leq j \leq s$. Then $\operatorname{dim}(Q \cap\{z=\lambda\}) \leq d-2$, for all $\lambda \in \mathbb{R}_{\geq 0}$. Moreover

1. if $\operatorname{dim}(Q) \leq d-2$, then $\operatorname{dim}(Q \cap\{z=\lambda\})=d-2$, at the most for one $\lambda \in \mathbb{R}_{\geq 0}$, i.e. in that case $\operatorname{dim}(Q)=\operatorname{dim}(Q \cap\{z=\lambda\})=d-2$ and $A(Q)=A(Q \cap\{z=\lambda\})$, i.e. $Q$ lies in the hyperplane $\{z=\lambda\}$.

2. If $\operatorname{dim}(Q)=d-1$, then $[A(Q) \cap\{z=m\}] \cap \mathbb{Z}^{d} \neq \phi$, for every $m \in \mathbb{Z}$.

3. If $\operatorname{dim}(Q \cap\{z=\lambda\})=d-2$, for some $\lambda \in \mathbb{R}_{>0}$, then $[A(n Q) \cap\{z=m\}] \cap \mathbb{Z}^{d} \neq \phi$, whenever $n, m \in \mathbb{Z}_{>0}$ such that $m / n=\lambda$.

Proof By definition, $Q \subseteq F$, for some $F \in F\left(F_{j}\right)$ or, for some $F \in F\left(C_{u}\right)$ and $u \in L\left(P_{D}\right)$. But such hyperplanes are transversal to the hyperplane $\{z=0\}$. Hence $\operatorname{dim} Q \cap\{z=\lambda\} \leq d-2$, for every $\lambda \in \mathbb{R}_{\geq 0}$.

(1) Suppose $\operatorname{dim}\left(Q \cap\left\{z=\lambda_{0}\right\}\right)=d-2$, for some $\lambda_{0} \in \mathbb{R}_{\geq 0}$. Then

$$
A(Q)=A\left(Q \cap\left\{z=\lambda_{0}\right\}\right)=A(Q) \cap\left\{z=\lambda_{0}\right\} .
$$

Therefore, $Q \subseteq A\left(Q \cap\left\{z=\lambda_{0}\right\}\right)$ and $Q \cap\{z=\lambda\}=\phi$, for $\lambda \neq \lambda_{0}$. Hence $\operatorname{dim} Q \cap\{z=\lambda\}=d-2$, at the most at one point.

(2) By Lemma 9, we have $Q \subseteq A(F)$, where $F \in F\left(F_{j}\right)$ or $F \in F\left(C_{u}\right)$.

Case (1) Let $F$ be a facet of $F_{j}$ for some $F_{j}$. Then $F \subseteq H_{i u}$ for some hyperplane $H_{i u}$ (as given in Notations 7) (5)) and $A(Q)=A(F)=H_{i u}$. Hence, for $m \in \mathbb{Z}$, we have $A(Q) \cap\{z=m\}=H_{i u} \cap\{z=m\}$, where it is easy to check that $m(u, 1) \in$ $H_{\text {iu }} \cap\{z=m\} \cap \mathbb{Z}^{d}$.

Case (2) If $F$ is a facet of $(u, 1)+C_{D}$, then $F=(u, 1)+F^{\prime}$, for some facet $F^{\prime}$ of $C_{D}$. Now $F^{\prime}$ is a cone over a facet $F^{\prime \prime}$ of $P_{D}$. Hence there exist a subset of vertices $\left\{u_{j}\right\} \subset \mathbb{Z}^{d-1}$ of $P_{D}$ such that $A(Q)=A(F)=\left\{(u, 1)+\sum_{j} \alpha_{j}\left(u_{j}, 1\right) \mid \alpha_{j} \in \mathbb{R}\right\}$. Now it is easy to check that $(u, 1)+(m-1)\left(u_{j}, 1\right) \in A(F) \cap\{z=m\} \cap \mathbb{Z}^{d}$.

(3) With the notations as in (2), we have $A(n Q \cap\{z=n \lambda\})=A(n Q) \cap\{z=$ $n \lambda\}=A(n F) \cap\{z=n \lambda\}$. Now, for $F \in F\left(F_{i}\right)$, one can check that $n \lambda(u, 1) \in$ $A(n F) \cap\{z=n \lambda\} \cap \mathbb{Z}^{d}$, and for $F \in F\left(C_{u}\right)$ for some $u \in L\left(P_{D}\right)$ one can check $n(u, 1)+(\lambda-1) n\left(u_{j}, 1\right) \in A(n F) \cap\{z=n \lambda\} \cap \mathbb{Z}^{d}$. This completes the proof of the lemma.

\section{Ehrhart quasi-polynomial for rational convex polytope}

In this section, we mainly deal with the Ehrhart's theory of lattice points inside rational convex polytopes. Recall that, if $\mathbb{Z}^{d}$ is the integral lattice in $d$-dimensional Euclidean space $\mathbb{R}^{d}$, then a convex polytope $P \subset \mathbb{R}^{d}$ is called integral (rational), if all its vertices have integral (rational) coordinates.

Definition 15 For a rational polytope $P$, the smallest number $\rho \in \mathbb{Q}_{>0}$ such that $\rho P$ is an integral polytope is called the rational denominator of $P$, and is denote by $\tau(P)$. 
Furthermore, the rational $i$-index $\tau_{i}(P)$ of $P$ is the smallest number $\rho \in \mathbb{Q}_{>0}$ such that for each $i$-dimensional face $F$ of $P$ the affine space $\rho A(F)$ contains integral points. Here $A(F)$ denotes the affine hull of $F$.

The following classical result is due to Ehrhart ([4]) and McMullen ([12]).

Theorem 16 Let $P \subset \mathbb{R}^{d}$ be a rational polytope. Then $i(P,-): \mathbb{Z}_{\geq 0} \rightarrow \mathbb{Z}_{\geq 0}$, given by

$$
i(P, n):=\#\left(n P \cap \mathbb{Z}^{d}\right)=\sum_{i=0}^{\operatorname{dim}(P)} C_{i}(P, n) n^{i}, \text { for } n \in \mathbb{Z}_{\geq 0},
$$

is a quasi-polynomial of degree $\operatorname{dim} P$, i.e., for every $i$, the coefficient $C_{i}(P, n)$ is periodic in $n$ of period $\tau_{i}(P)$, and $C_{\operatorname{dim} P}(P, n)$ is not identically zero (in fact is $=\mathrm{rVol}_{\operatorname{dim} P}(P)$, if $A(P)$ contains an integral point $)$.

Moreover if $P^{\circ}$ denotes the interior of $P$ in the affine span of $P$, then $i\left(P^{\circ}, n\right)=$ $\#\left(n P^{\circ} \cap \mathbb{Z}^{d}\right)=(-1)^{\operatorname{dim}(P)} i(P,-n)$. In particular, $A(F) \cap \mathbb{Z}^{d} \neq \phi$, for every $F \in$ $F(P)$ implies

$$
C_{\operatorname{dim}(P)-1}(P, n)=(1 / 2) \sum_{F \in F(P)} \mathrm{rVol}_{\operatorname{dim}(P)-1}(F) .
$$

Here note that, since $i(P, n)$ is a quasi-polynomial, it can be defined for all $n \in \mathbb{Z}$. McMullen has generalised Theorem 16 for the rational Minkowski sum of finitely many polytopes $P_{1}, \ldots, P_{k} \subset \mathbb{R}^{d}$. Throughout this section, we use the following notations and definition from the literature.

Notations 17 1. For $\mathbf{r}=\left(r_{1}, \ldots, r_{k}\right) \in \mathbb{R}^{k}$ and $\mathbf{l}=\left(l_{1}, \ldots, l_{k}\right) \in \mathbb{Z}_{\geq 0}^{k}$, we denote $\prod_{i=1}^{k} r_{i}^{l_{i}}$ by $\mathbf{r}^{\mathbf{l}}$ and $\sum_{i} l_{i}$ by $|\mathbf{I}|$.

2. The Hadamard product $\mathbf{r} \odot \mathbf{s}$ of two rational vectors $\mathbf{r}, \mathbf{s} \in \mathbb{Q}^{k}$ is the coordinate-wise product $\mathbf{r} \odot \mathbf{s}=\left(r_{1} s_{1}, \ldots, r_{k} s_{k}\right)$.

With these notations, McMullen's result (see comments on page 2 of [7]) on the Ehrhart quasi-polynomial of a Minkowski sum of rational polytopes can be stated as follows.

Theorem 18 Let $P_{1}, \ldots, P_{k} \subset \mathbb{R}^{d}$ be rational polytopes. Then the function $Q\left(P_{1}, \ldots, P_{k},-\right): \mathbb{Q}_{\geq 0}^{k} \rightarrow \mathbb{N}$ given by

$$
Q\left(P_{1}, \ldots, P_{k}, \mathbf{r}\right)=\#\left(\sum_{i} r_{i} P_{i} \cap \mathbb{Z}^{d}\right), \text { for } \boldsymbol{r}=\left(r_{1}, \ldots, r_{k}\right) \in \mathbb{Q}_{\geq 0}^{k}
$$

is a rational quasi-polynomial of degree $\operatorname{dim}\left(P_{1}+\cdots+P_{k}\right)$ with period $\tau=$ $\left(\tau\left(P_{1}\right), \ldots, \tau\left(P_{k}\right)\right)$, i.e., $Q\left(P_{1}, \ldots, P_{k}, \mathbf{r}\right)=\sum_{|\boldsymbol{l}| \leq d} p_{\boldsymbol{l}}(\boldsymbol{r}) \boldsymbol{r} \boldsymbol{l}$, where $p_{\boldsymbol{l}}: \mathbb{Q}_{\geq 0}^{k} \rightarrow \mathbb{Q}$ is a periodic function with period $\tau_{i}=\tau\left(P_{i}\right)$ in the $i$ th argument, $i=1, \ldots, k$, and $p_{\boldsymbol{l}}(\mathbf{r})$ is nonzero positive constant for some $\boldsymbol{l} \in \mathbb{Z}_{\geq 0}^{k}$ with $|\boldsymbol{l}|=\operatorname{dim}\left(P_{1}+\cdots+P_{k}\right)$. 
Proof See Theorem 7 of [12].

Definition $19 Q\left(P_{1}, \ldots, P_{k},-\right)$ is called the rational Ehrhart quasi-polynomial of the rational polytopes $P_{1}, \ldots, P_{k}$, and the $\mathbf{l t h}$ coefficient of $Q\left(P_{1}, \ldots, P_{k},-\right)$ is denoted by $Q_{\mathbf{l}}\left(P_{1}, \ldots, P_{k},-\right)$.

In 2011, Linke has proved (see Theorem 1.2, Corollary 1.5 and Theorem 1.6 of [11]) the following result about the coefficients of rational Ehrhart quasi-polynomial of a rational polytope.

Theorem 20 Let $P \subset \mathbb{R}^{d}$ be a rational polytope of dimension d with rational Ehrhart quasi-polynomial

$$
i(P, r):=\#\left(r P \cap \mathbb{Z}^{d}\right)=\sum_{i=0}^{\operatorname{dim}(P)} C_{i}(P, r) r^{i}, \text { where } r \in \mathbb{Q}_{\geq 0} .
$$

Then (1) there exist $0=r_{0}<r_{1}<\cdots<r_{l}=\tau(P)$, such that $C_{i}(P,-)$ is a polynomial of degree $d-i$ on $\left(r_{m-1}, r_{m}\right)$, for each $m=1, \ldots, l$ and $i=0, \ldots, d$. (2) The reciprocity theorem is true for rational dilates and for all dimension, i.e. for all $r \in \mathbb{Q}_{\geq 0}, i(P, r)=(-1)^{\operatorname{dim}(P)} i(P,-r)$. In particular $C_{d}(P, r)=\operatorname{Vol}_{d}(P)$, for all $r \in \mathbb{Q}_{>0}$, and, in addition, if $C_{d-1}(P, r)$ is independent of $r>0$, then $C_{d-1}(P, r)=(1 / 2) \sum_{F \in F(P)} \mathrm{rVol}_{d-1}(F)$.

Later, the above theorem was generalised for Minkowski sum of polytopes by Henk and Linke in their paper [7, Theorem 1.3].

We recall briefly some important points relevant to the statement of Theorem 1.3 of [7].

For a polytope $P \subset \mathbb{R}^{d}$, let $h(P,-): \mathbb{R}^{d} \rightarrow \mathbb{R}$ be its support function, i.e. $h(P, v)=\max \{\langle v, x\rangle: x \in P\}$. A hyperplane $H(P, v):=\left\{x \in \mathbb{R}^{d} \mid\langle x, v\rangle=\right.$ $h(P, v)\}$, for $v \in \mathbb{R}^{d} \backslash\{0\}$ is called a supporting hyperplane of $P$. If $P$ is fulldimensional, i.e. $\operatorname{dim}(P)=d$, then each facet $F$ of $P$ is given by a unique supporting hyperplane $H_{F}=\left\{x \in \mathbb{R}^{d} \mid\left\langle x, a_{F}\right\rangle=b_{F}\right\}$, where $\left(a_{F}, b_{F}\right) \in \mathbb{R}^{d} \times \mathbb{R}$ is unique up to multiplication by a positive real number. Let $H_{F}^{-}=\left\{x \in \mathbb{R}^{d} \mid\left\langle x, a_{F}\right\rangle \leq b_{F}\right\}$, Let $P \subset \mathbb{R}^{d}$ be a full-dimensional lattice polytope; the hyperplane representation of $P$ is

$$
P=\bigcap_{F \in F(P)} H_{F}^{-}=\bigcap_{F \in F(P)}\left\{x \in \mathbb{R}^{d} \mid\left\langle x, a_{F}\right\rangle \leq b_{F}\right\}
$$

where the intersection runs over all facets $F$ of $P$. We call $a_{F} /\left\|a_{F}\right\|$ the outer unit normal of the facet $F$ of $P$.

Let $P_{1}, \ldots, P_{k} \subset \mathbb{R}^{d}$ be rational polytopes with $\operatorname{dim}\left(P_{1}+\cdots+P_{k}\right)=d$. Let $\mathbf{v}_{1}, \ldots, \mathbf{v}_{m} \in \mathbb{Z}^{d}$, be the outer normals of the facets of the rational polytope $P_{1}+$ $\ldots+P_{k}$. Observe that for all $\mathbf{r} \in \mathbb{R}_{>0}^{k}$ the facets of the polytope $r_{1} P_{1}+\ldots+r_{k} P_{k}$ have the same outer normals $\mathbf{v}_{1}, \ldots, \mathbf{v}_{m}$. For details about support function and face decomposition of Minkowski sum, see [15]. Now, for $\mathbf{r} \in \mathbb{R}_{>0}^{k}$ and $\mathbf{z} \in \mathbb{Z}^{d}$ we know $\mathbf{z} \in \sum_{i=1}^{k} r_{i} P_{i}$ if and only if $\left\langle\mathbf{z}, \mathbf{v}_{j}\right\rangle \leq \sum_{i=1}^{k} r_{i} h\left(P_{i}, \mathbf{v}_{j}\right)$ for $1 \leq j \leq m$. Thus 
$Q\left(P_{1}, \ldots, P_{k}, \mathbf{r}\right)$ is a constant function on the interior of the $k$-dimensional cells induced by the hyperplane arrangement

$$
\left\{\left\{\mathbf{r} \in \mathbb{R}_{>0}^{k}: \sum_{i=1}^{k} r_{i} h\left(P_{i}, \mathbf{v}_{j}\right)=\left\langle\mathbf{z}, \mathbf{v}_{j}\right\rangle\right\}: \mathbf{z} \in \mathbb{Z}^{d}, j=1, \ldots, m\right\} .
$$

Let $S$ be the interior of a fixed $k$-dimensional cell given by this section. Then $Q\left(P_{1}, \ldots, P_{k},-\right)$ is constant on $S$.

The result of Henk-Linke (Theorem 1.3) can be stated as follows:

Theorem 21 Let $P_{1}, \ldots, P_{k} \subset \mathbb{R}^{d}$ be rational polytopes with $\operatorname{dim}\left(P_{1}+\cdots+P_{k}\right)=d$ and let $\boldsymbol{l} \in \mathbb{Z}_{\geq 0}^{k}$ with $|\boldsymbol{l}| \leq d$. Then $Q_{l}\left(P_{1}, \ldots, P_{k},-\right)$ is a piecewise polynomial function of degree at most $d-|\boldsymbol{l}|$ on open $k$-cells given by the hyperplane arrangements as in (7).

Here we consider the case with $k=2$. By Theorem $18, Q\left(P_{1}, P_{2},-\right)$ is a quasi-polynomial of degree $\operatorname{dim}\left(P_{1}+P_{2}\right)$ with period $\tau=\left(\tau_{1}, \tau_{2}\right)$. The hyperplane arrangement in (7) can be rewritten as

$$
\left\{\left\{\mathbf{r} \in \mathbb{R}_{>0}^{2}: r_{1} h\left(P_{1}, \mathbf{v}_{j}\right)+r_{2} h\left(P_{2}, \mathbf{v}_{j}\right)=\left\langle\mathbf{z}, \mathbf{v}_{j}\right\rangle\right\}: \mathbf{z} \in \mathbb{Z}^{d}, j=1, \ldots, m\right\}
$$

and $Q\left(P_{1}, P_{2},-\right)$ is constant on each open 2-cell in the complement of these lines.

Notations 221 . For $\mathbf{z} \in \mathbb{Z}^{d}$, and $1 \leq j \leq m$, we denote the line

$$
L_{j}(\mathbf{z})=\left\{\mathbf{r} \in \mathbb{R}^{2}: r_{1} h\left(P_{1}, \mathbf{v}_{j}\right)+r_{2} h\left(P_{2}, \mathbf{v}_{j}\right)=\left\langle\mathbf{z}, \mathbf{v}_{j}\right\rangle\right\} .
$$

the positive halfspace $L_{j}(\mathbf{z})^{+}=\left\{\mathbf{r} \in \mathbb{R}^{2}: r_{1} h\left(P_{1}, \mathbf{v}_{j}\right)+r_{2} h\left(P_{2}, \mathbf{v}_{j}\right) \geq\left\langle\mathbf{z}, \mathbf{v}_{j}\right\rangle\right\}$ and the positive open halfspace $L_{j}(\mathbf{z})^{+\circ}$ the interior of $L_{j}(\mathbf{z})^{+}$, i.e.,

$$
L_{j}(\mathbf{z})^{+\circ}=\left\{\mathbf{r} \in \mathbb{R}^{2}: r_{1} h\left(P_{1}, \mathbf{v}_{j}\right)+r_{2} h\left(P_{2}, \mathbf{v}_{j}\right)>\left\langle\mathbf{z}, \mathbf{v}_{j}\right\rangle\right\} .
$$

Similarly, one defines $L_{j}(\mathbf{z})^{-}$and $L_{j}(\mathbf{z})^{-\circ}$ for $\mathbf{z} \in \mathbb{Z}^{d}$ and for $j=1, \ldots, m$.

2. Denote the period rectangle $T=\left(0, \tau_{1}\right] \times\left(0, \tau_{2}\right]$, where $\left(\tau_{1}, \tau_{2}\right)=\left(\tau\left(P_{1}\right), \tau\left(P_{2}\right)\right)$ $\in \mathbb{Q}_{>0}^{2}$.

3. Note that for each $j \in\{1, \ldots, m\}$, there can be only finitely many $L_{j}(\mathbf{z})$ intersecting the period rectangle $T$, as $\mathbf{v}_{j} \in \mathbb{Z}^{d}$.

4. $\mathbb{R}_{>0}^{2}$ is the disjoint union of locally closed sets, namely

$$
\mathbb{R}_{>0}^{2}=\left(\bigcup_{S \in \tilde{C}_{P}} S\right) \cup\left(\bigcup_{I \in \tilde{I}_{P}} I\right) \cup T_{0},
$$

where $\tilde{C}_{P}=$ the set of open 2-cells obtained by the hyperplane arrangement as given in (8), the set

$$
T_{0}=\left\{L_{j}(\mathbf{z}) \cap L_{i}\left(\mathbf{z}^{\prime}\right) \cap \mathbb{R}_{>0}^{2} \mid L_{j}(\mathbf{z}) \neq L_{i}\left(\mathbf{z}^{\prime}\right), \quad \text { for all } z, z^{\prime} \in \mathbb{Z}^{d} \text { and } 1 \leq i, j \leq m\right\}
$$


is a discrete set of points and

$$
\tilde{I}_{P}=\text { the connected components of } \mathbb{R}_{>0}^{2} \backslash\left[\left(\bigcup_{S \in \tilde{C}_{P}} S\right) \cup T_{0}\right]
$$

is the set of open intervals. In particular, for any $I \in \tilde{I}_{P}$, there exists a unique line $L_{j_{0}}\left(\mathbf{z}_{0}\right)$ such that $I$ is a connected component of

$$
\left[L_{j_{0}}\left(\mathbf{z}_{0}\right) \backslash\left\{L_{j_{0}}\left(\mathbf{z}_{0}\right) \cap L_{j}(\mathbf{z}) \mid L_{j_{0}}\left(\mathbf{z}_{0}\right) \neq L_{j}(\mathbf{z}), \quad 1 \leq j \leq m, \quad \mathbf{z} \in \mathbb{Z}^{d}\right\}\right] \cap \mathbb{R}_{>0}^{2} .
$$

Definition 23 For given $I \in \tilde{I}_{P}$, we associate (the unique) $S_{I} \in \tilde{C}_{P}$ as follows: By definition $I \subset L_{j}(\mathbf{z})$, for a unique line $L_{j}(\mathbf{z})$ (as in Notation 22) in $\mathbb{R}^{2}$. Then $S_{I}$ is the unique cell in $\tilde{C}_{P}$ such that $I \subset \bar{S}_{I}$, the closure of $S_{I}$ in $\mathbb{R}_{>0}^{2}$ and $S_{I} \subset L_{j}(\mathbf{z})^{+o}$.

Lemma 24 Given $\left(I, S_{I}\right) \in \tilde{I}_{P} \times \tilde{C}_{P}$, we have

$$
S_{I} \subset L_{j_{l}}\left(\mathbf{z}_{i_{l}}\right)^{+} \Longleftrightarrow I \subset L_{j_{l}}\left(\mathbf{z}_{i_{l}}\right)^{+}
$$

Moreover, if $L_{j_{0}}\left(\mathbf{z}_{i_{0}}\right)$ is the line containing $I$, then for $L_{j_{l}}\left(\mathbf{z}_{i_{l}}\right) \neq L_{j_{0}}\left(\mathbf{z}_{i_{0}}\right)$, we have

$$
S_{I} \subset L_{j_{l}}\left(\mathbf{z}_{i_{l}}\right)^{+} \Longleftrightarrow S_{I} \subset L_{j_{l}}\left(\mathbf{z}_{i_{l}}\right)^{+o} \Longleftrightarrow I \subset L_{j_{l}}\left(\mathbf{z}_{i_{l}}\right)^{+o} \Longleftrightarrow I \subset L_{j_{l}}\left(\mathbf{z}_{i_{l}}\right)^{+} .
$$

Proof It is easy to check.

Lemma 25 Given $S \in \tilde{C}_{P}, Q\left(P_{1}, P_{2}, s\right)=$ constant for all $s \in S$.

Given $\left(I, S_{I}\right) \in \tilde{I}_{P} \times \tilde{C}_{P}$

$$
Q\left(P_{1}, P_{2}, s\right)=\text { constant for all } s \in S_{I} \cup I \text {. }
$$

Proof It is easy to check.

Lemma 26 Let $\mathbf{u}=\left(u_{1}, u_{2}\right) \in \mathbb{Z}_{\geq 0}^{2}$ and let $T=\left(0 \times \tau_{1}\right] \times\left(0 \times \tau_{2}\right]$ be as in Notations 22. Then,

1. for given $S \in \tilde{C}_{P}$, we have $Q\left(P_{1}, P_{2}, \boldsymbol{s}\right)=$ constant, for all $\boldsymbol{s} \in S \cap T+\boldsymbol{u} \odot \tau$ and,

2. for given $\left(I, S_{I}\right) \in \tilde{I}_{P} \times \tilde{C}_{P}$, we have $Q\left(P_{1}, P_{2}, s\right)=$ constant, for all $s \in$ $\left(S_{I} \cup I\right) \cap T+\boldsymbol{u} \odot \tau$.

Proof For $\left(u_{1}, u_{2}\right) \in \mathbb{Z}_{\geq 0}^{2}$, the polytope $u_{1} \tau_{1} P_{1}+u_{2} \tau_{2} P_{2}$ is an integral polytope. Therefore, for every facet $F_{j}$ of (this polytope) with the outer normal $\mathbf{v}_{j}$, we can choose $\tilde{\mathbf{z}}_{j} \in F_{j} \cap \mathbb{Z}^{d}$ such that $h\left(u_{1} \tau_{1} P_{1}+u_{2} \tau_{2} P_{2}, \mathbf{v}_{j}\right)=\left\langle\tilde{\mathbf{z}}_{j}, \mathbf{v}_{j}\right\rangle$. Now, it is easy to check that, for every $1 \leq j \leq m$,

$$
L_{j}\left(\mathbf{z}+\tilde{\mathbf{z}}_{j}\right)=L_{j}(\mathbf{z})+\mathbf{u} \odot \tau, \quad L_{j}\left(\mathbf{z}+\tilde{\mathbf{z}}_{j}\right)^{+o}=L_{j}(\mathbf{z})^{+o}+\mathbf{u} \odot \tau
$$


and

$$
L_{j}\left(\mathbf{z}+\tilde{\mathbf{z}}_{j}\right)^{-o}=L_{j}(\mathbf{z})^{-o}+\mathbf{u} \odot \tau
$$

A given $S \in \tilde{C}_{P}$ can be written as

$$
S=\left[\cap_{\mu=0}^{s_{1}} L_{j_{\mu}}\left(\mathbf{z}_{i_{\mu}}\right)^{+\circ}\right] \cap\left[\cap_{\nu=1}^{s_{2}} L_{l_{\nu}}\left(\mathbf{z}_{k_{\nu}}\right)^{-\circ}\right] \cap \mathbb{R}_{>0}^{2}
$$

for some $\mathbf{z}_{i_{\mu}}, \mathbf{z}_{k_{v}} \in \mathbb{Z}^{d}$ and $1 \leq j_{\mu}, l_{v} \leq m$.

Therefore,

$$
\begin{aligned}
S+\mathbf{u} \odot \tau & =\left[\cap_{\mu=0}^{s_{1}} L_{j_{\mu}}\left(\mathbf{z}_{i_{\mu}}\right)^{+\circ}+\mathbf{u} \odot \tau\right] \cap\left[\cap_{\nu=1}^{s_{2}} L_{l_{v}}\left(\mathbf{z}_{k_{v}}\right)^{-\circ}+\mathbf{u} \odot \tau\right] \cap\left[\mathbb{R}_{>0}^{2}+\mathbf{u} \odot \tau\right], \\
& =\left[\cap_{\mu=0}^{s_{1}} L_{j_{\mu}}\left(\mathbf{z}_{i_{\mu}}+\tilde{\mathbf{z}}_{j_{\mu}}\right)^{+\circ}\right] \cap\left[\cap_{\nu=1}^{s_{2}} L_{l_{v}}\left(\mathbf{z}_{k_{\nu}}+\tilde{\mathbf{z}}_{l_{\nu}}\right)^{-\circ}\right] \cap\left[\mathbb{R}_{>0}^{2}+\mathbf{u} \odot \tau\right] .
\end{aligned}
$$

Note that, for any $\mathbf{z} \in \mathbb{Z}^{d}$ and $1 \leq j \leq m$, if $S \in \tilde{C}_{P}$, then we have $L_{j}\left(\mathbf{z}-\tilde{\mathbf{z}}_{j}\right) \cap S=\phi$, since $L_{j}\left(\mathbf{z}-\tilde{\mathbf{z}}_{j}\right)$ is one of the lines in the hyperplane arrangement 7 . This implies $L_{j}(\mathbf{z}) \cap(S+\mathbf{u} \odot \tau)=\left(L_{j}\left(\mathbf{z}-\tilde{\mathbf{z}}_{j}\right) \cap S\right)+\mathbf{u} \odot \tau=\phi$. Hence $S+\mathbf{u} \odot \tau \subseteq S_{1}$, for some $S_{1} \in \tilde{C}_{P}$.

Therefore, by Lemma 25,

$Q\left(P_{1}, P_{2}, \mathbf{s}\right)=$ constant for all $\mathbf{s} \in(S+\mathbf{u} \odot \tau) \cap(T+\mathbf{u} \odot \tau)=(S \cap T)+\mathbf{u} \odot \tau$.

This proves Assertion (1).

Note that $I \in \tilde{I}_{P}$ if and only if $I \subset L_{j_{0}}\left(\mathbf{z}_{0}\right)$, some $1 \leq j_{0} \leq m$ and $\mathbf{z}_{0} \in \mathbb{Z}^{d}$, such that $I$ is a connected component of

$$
\left(L_{j_{0}}\left(\mathbf{z}_{0}\right) \backslash\left\{L_{j_{0}}\left(\mathbf{z}_{0}\right) \cap L_{j}(\mathbf{z}) \mid \mathbf{z} \in \mathbb{Z}^{d}, \quad 1 \leq j \leq m \text { and } L_{j}(\mathbf{z}) \neq L_{j_{0}}\left(\mathbf{z}_{0}\right)\right\}\right) \cap \mathbb{R}_{>0}^{2} .
$$

This implies that $I+\mathbf{u} \odot \tau$ is a connected component of

$$
\left[\left\{L _ { j _ { 0 } } ( \mathbf { z } _ { 0 } + \tilde { \mathbf { z } } _ { j _ { 0 } } ) \backslash \left\{L_{j_{0}}\left(\mathbf{z}_{0}+\tilde{\mathbf{z}}_{j_{0}}\right) \cap L_{j}\left(\mathbf{z}+\tilde{\mathbf{z}}_{j}\right) \mid \mathbf{z} \in \mathbb{Z}^{d}, \quad 1 \leq j \leq m,\right.\right.\right.
$$

and

$$
\left.\left.L_{j_{0}}\left(\mathbf{z}_{0}+\tilde{\mathbf{z}}_{j_{0}}\right) \neq L_{j}\left(\mathbf{z}+\tilde{\mathbf{z}}_{j}\right)\right\}\right] \cap \mathbb{R}_{>0}^{2} .
$$

Hence $I+\mathbf{u} \odot \tau \in \tilde{I}_{P}$. One can easily check (from Lemma 24) that $S_{I}+\mathbf{u} \odot \tau=$ $S_{I+\mathbf{u} \odot \tau}$. Hence,

$$
\left(S_{I+\mathbf{u} \odot \tau} \cup(I+\mathbf{u} \odot \tau)\right) \cap(T+\mathbf{u} \odot \tau)=\left(\left(S_{I} \cup I\right) \cap T\right)+\mathbf{u} \odot \tau .
$$

Now, by Lemma 25,

$$
Q\left(P_{1}, P_{2}, \mathbf{s}\right)=\text { constant for all } \mathbf{s} \in\left(\left(S_{I} \cup I\right) \cap T\right)+\mathbf{u} \odot \tau \text {. }
$$


This completes the proof of the lemma.

In the proof of the next lemma, we imitate the arguments given in the proof of Lemma 2.2 and Lemma 2.3 of [7].

Lemma 27 Let $p: \mathbb{Q}_{>0}^{2} \longrightarrow \mathbb{Q}$ be a rational quasi-polynomial of degree $n \geq 1$ with period $\tau=\left(\tau_{1}, \tau_{2}\right) \in \mathbb{Q}_{>0}^{2}$ and constant leading coefficients, i.e.,

$$
p(\mathbf{r})=\sum_{l_{1}+l_{2} \leq n} p_{l_{1}, l_{2}}(\mathbf{r}) r_{1}^{l_{1}} r_{2}^{l_{2}}, \text { where } \mathbf{r}=\left(r_{1}, r_{2}\right) \in \mathbb{Q}_{>0}^{2} \text {, }
$$

such that

1. $p_{l_{1}, l_{2}}(\mathbf{r}) \in \mathbb{Q}$ is constant for all $\left(l_{1}, l_{2}\right) \in \mathbb{Z}_{\geq 0}^{2}$ with $l_{1}+l_{2}=n$, and for every $\mathbf{r} \in \mathbb{Q}_{>0}^{2}$,

2. $p_{l_{1}, l_{2}}: \mathbb{Q}_{>0}^{2} \longrightarrow \mathbb{Q}$ are periodic functions with period $\tau=\left(\tau_{1}, \tau_{2}\right)$ for all $\left(l_{1}, l_{2}\right) \in \mathbb{Z}_{\geq 0}^{2}$ with $l_{1}+l_{2}<n$.

Let $E \subseteq \mathbb{R}_{>0}^{2}$ be a subset of $\mathbb{R}^{2}$, such that, for every $\mathbf{u} \in \mathbb{Z}_{\geq 0}^{2}$, there exists $c_{\mathbf{u}} \in \mathbb{Q}$ with

$$
p(\mathbf{r}+\mathbf{u} \odot \tau)=c_{\mathbf{u}} \text { for all } \mathbf{r} \in E \cap \mathbb{Q}^{2} .
$$

Then for all $\left(l_{1}, l_{2}\right) \in \mathbb{Z}_{\geq 0}^{2}$ with $l_{1}+l_{2} \leq n$, the coefficient function $p_{l_{1}, l_{2}}: E \cap \mathbb{Q}_{>0}^{2} \longrightarrow$ $\mathbb{Q}$ is a polynomial of degree at most $n-\left(l_{1}+l_{2}\right)$.

Proof We prove the lemma by induction on $\operatorname{deg}(p)=n$. For $n=1$ and for $\mathbf{r} \in E \cap \mathbb{Q}^{2}$, we have

$$
c_{\mathbf{0}}=p(\mathbf{r})=p_{0,0}(\mathbf{r})+p_{(1,0)}(\mathbf{r}) r_{1}+p_{(0,1)}(\mathbf{r}) r_{2}
$$

Therefore, $p_{0,0}: E \cap \mathbb{Q}^{2} \longrightarrow \mathbb{Q}$ is a polynomial of degree $\leq 1$ (if $p_{(1,0)}=p_{(0,1)}=0$ then $p_{(0,0)}=$ constant).

Now let $n \geq 2$.

Let

$$
q(\mathbf{r})=p\left(\mathbf{r}+\mathbf{e}_{2} \odot \tau\right)-p(\mathbf{r})=p\left(r_{1}, r_{2}+\tau_{2}\right)-p\left(r_{1}, r_{2}\right)
$$

Then, for $\mathbf{r} \in E \cap \mathbb{Q}_{>0}^{2}$, and $\mathbf{u} \in \mathbb{Z}_{\geq 0}^{2}$, we have

$$
q(\mathbf{r}+\mathbf{u} \odot \tau)=p\left(\mathbf{r}+\left(\mathbf{u}+\mathbf{e}_{2}\right) \odot \tau\right)-p(\mathbf{r}+\mathbf{u} \odot \tau)=c_{\mathbf{u}+\mathbf{e}_{2}}-c_{\mathbf{u}} .
$$

Next we show that $q$ is a quasi-polynomial of degree $n-1$ and of period $\tau$ with constant leading coefficients. Now, for every $\mathbf{r} \in \mathbb{Q}_{\geq 0}^{2}$,

$$
\begin{aligned}
q(\mathbf{r}) & =p\left(r_{1}, r_{2}+\tau_{2}\right)-p\left(r_{1}, r_{2}\right) \\
& =\sum_{l_{2} \neq 0, l_{1}+l_{2} \leq n} p_{l_{1}, l_{2}}(\mathbf{r}) r_{1}^{l_{1}}\left[\tau_{2}^{l_{2}}+\left(\begin{array}{c}
l_{2} \\
1
\end{array}\right) \tau_{2}^{l_{2}-1} r_{2}+\cdots+\left(\begin{array}{c}
l_{2} \\
l_{2}-1
\end{array}\right) \tau_{2} r_{2}^{l_{2}-1}\right]
\end{aligned}
$$




$$
=\sum_{l_{1}, l_{2} \geq 0,} q_{l_{1}+l_{2} \leq n-1} l_{2}(\mathbf{r}) r_{1}^{l_{1}} r_{2}^{l_{2}}
$$

where

$q_{l_{1}, l_{2}}(\mathbf{r})=p_{l_{1}, l_{2}+1}(\mathbf{r}) c_{1}\left(l_{1}, l_{2}\right)+p_{l_{1}, l_{2}+2}(\mathbf{r}) c_{2}\left(l_{1}, l_{2}\right)+\cdots+p_{l_{1}, n-l_{1}}(\mathbf{r}) c_{n-l_{1}-l_{2}}\left(l_{1}, l_{2}\right)$

and $c_{i}\left(l_{1}, l_{2}\right)$ are positive constants. Therefore, $q_{l_{1}, l_{2}}: \mathbb{Q}_{>0}^{2} \longrightarrow Q$ are periodic functions with period $\left(\tau_{1}, \tau_{2}\right)$ and, for every $\mathbf{r} \in \mathbb{Q}_{>0}^{2}$,

$q_{l_{1}, n-1-l_{1}}(\mathbf{r})=p_{l_{1}, n-l_{1}}(\mathbf{r}) c_{1}\left(l_{1}, l_{2}\right)=C_{l_{1}}=$ a constant, for every $0 \leq l_{1} \leq n-1$.

Therefore, by induction hypothesis, $q_{l_{1}, l_{2}}: E \cap \mathbb{Q}_{>0}^{2} \longrightarrow \mathbb{Q}$ is a polynomial of degree at most $n-1-l_{1}-l_{2}$, for all $\left(l_{1}, l_{2}\right) \in \mathbb{Z}_{\geq 0}^{2}$ such that $l_{1}+l_{2} \leq n-1$. By (13) and descending induction on $\left(l_{1}, l_{2}\right)$, we deduce that $p_{l_{1}, l_{2}+1}(\mathbf{r}): E \cap \mathbb{Q}^{2} \longrightarrow \mathbb{Q}$ is a polynomial of degree at most $n-\left(l_{1}+l_{2}+1\right)$. Similarly, by considering the function $q^{\prime}\left(r_{1}, r_{2}\right)=p\left(r_{1}+\tau_{1}, r_{2}\right)-p\left(r_{1}, r_{2}\right)$, we deduce that $p_{l_{1}+1, l_{2}}(\mathbf{r}): E \longrightarrow \mathbb{Q}$ is a polynomial of degree at most $n-\left(l_{1}+l_{2}+1\right)$. Now, the function $p_{0,0}: \mathbb{Q}_{\geq 0}^{2} \longrightarrow \mathbb{Q}$ is given by

$$
p_{0,0}(\mathbf{r})=c_{0}-\sum_{(0,0) \neq\left(l_{1}, l_{2}\right) \in \mathbb{Z}_{\geq 0}^{2}, l_{1}+l_{1} \leq n} p_{l_{1}, l_{2}}(\mathbf{r}) r_{1}^{l_{1}} r_{2}^{l_{2}}
$$

and hence $p_{0,0}: E \cap \mathbb{Q}_{>0}^{2} \longrightarrow \mathbb{Q}$ is a polynomial of degree at most $n$. This completes the proof of the lemma.

Theorem 28 Let $P_{1}, P_{2} \subset \mathbb{R}^{d}$ be two rational convex polytopes such that $\operatorname{dim}\left(P_{1}+P_{2}\right)$ $=d$. Let $Q\left(P_{1}, P_{2} ;-\right): \mathbb{Q}_{\geq 0}^{2} \longrightarrow \mathbb{Q}$ be the rational quasi-polynomial of degree $d$, given by

$$
Q\left(P_{1}, P_{2}, ; \mathbf{r}\right)=\#\left(\left(r_{1} P_{1}+r_{2} P_{2}\right) \cap \mathbb{Z}^{d}\right)=\sum_{\left(l_{1}, l_{2}\right) \in \mathbb{Z}_{\geq 0}^{2}} p_{l_{1}, l_{2}}(\mathbf{r}) r_{1}^{l_{1}} r_{2}^{l_{2}}
$$

Then there is a finite set $\tilde{S}$ consisting of locally closed, bounded subsets of $\mathbb{R}_{>0}^{2}$, and a finite set of polynomials

$$
\left\{f_{l_{1}, l_{2}}^{U}: U \longrightarrow \mathbb{Q} \mid U \in \tilde{S}, \quad\left(l_{1}, l_{2}\right) \in \mathbb{Z}_{\geq 0}^{2}, \quad l_{1}+l_{2} \leq n\right\}
$$

such that

1. $\mathbb{R}_{>0}^{2}=\cup_{U \in \tilde{S}} \cup_{\mathbf{u} \in \mathbb{Z}_{\geq 0}^{2}}(U+\mathbf{u} \odot \tau)$ and

$$
p_{l_{1}, l_{2}}(\mathbf{r})=f_{l_{1}, l_{2}}^{U}(\mathbf{r}), \quad \text { for every } \quad \mathbf{r} \in U \in \tilde{S} \text {. }
$$


2. In particular, there exist non-negative constants $C_{l_{1}, l_{2}}$ such that for all $\mathbf{r} \in \mathbb{Q}_{>0}^{2}$,

$$
\left|p_{l_{1}, l_{2}}(\mathbf{r})\right| \leq C_{l_{1}, l_{2}} \text {, and } p_{l_{1}, d-l_{1}}(\mathbf{r})=C_{l_{1}, d-l_{1}} .
$$

Proof Let

$$
\tilde{S}=\left\{\left(S_{I} \cup I\right) \cap T \mid S_{I} \in \tilde{C}_{P}, \quad I \in \tilde{I}_{P} \quad\left(S_{I} \cup I\right) \cap T \neq \phi\right\} \cup\left\{T_{0} \cap T\right\} .
$$

Since $T_{0}$ is a discrete set of points, the set $T_{0} \cap T=$ a finite set of points.

By Lemmas 26 and 27, for every $U \in \tilde{S}$ there is a set of polynomials,

$$
\left\{f_{l_{1}, l_{2}}^{U}: U \longrightarrow \mathbb{Q} \mid \quad\left(l_{1}, l_{2}\right) \in \mathbb{Z}_{\geq 0}^{2}, \quad l_{1}+l_{2} \leq n\right\}
$$

such that $p_{l_{1}, l_{2}}(\mathbf{r})=f_{l_{1}, l_{2}}^{U}(\mathbf{r})$, for every $\mathbf{r} \in U$.

Now since each $p_{l_{1}, l_{2}}: \mathbb{Q}_{>0}^{2} \longrightarrow \mathbb{Q}$ is a periodic function of period $\tau=\left(\tau_{1}, \tau_{2}\right)$, we can choose $C_{l_{1}, l_{2}}=\max \left\{\left|f_{l_{1}, l_{2}}^{U}(\mathbf{r})\right| \mid \mathbf{r} \in U, U \in \tilde{S}\right\}$. This proves the theorem.

Remark 29 Theorem 28 can be generalised to the Minkowski sum of any finite number of polytopes, say $P_{1}, P_{2}, \ldots, P_{n}$ in $\mathbb{R}^{d}$ where $\operatorname{dim}\left(P_{1}+\cdots+P_{n}\right)=d$ : For this we express $\mathbb{R}_{>0}^{d}$ as the disjoint union of locally closed sets, namely

$$
\mathbb{R}_{>0}^{d}=\left(\bigcup_{S \in \tilde{C}_{n}} S\right) \cup\left(\bigcup_{S \in \tilde{C}_{n-1}} S\right) \cup \cdots\left(\bigcup_{S \in \tilde{C}_{1}} S\right) \cup S_{0},
$$

where $\tilde{C}_{k}$ denotes the set of $k$-cells obtained by the hyperplane arrangements as given in (7). For $\mathbf{z} \in \mathbb{Z}^{d}$, and $1 \leq j \leq m$, let $H_{j}$ (z) denote the hyperplane

$$
H_{j}(\mathbf{z})=\left\{\mathbf{r} \in \mathbb{R}^{n}: \sum_{i=1}^{n} r_{i} h\left(P_{i}, \mathbf{v}_{j}\right)=\left\langle\mathbf{z}, \mathbf{v}_{j}\right\rangle\right\}
$$

where $\mathbf{v}_{1}, \ldots, \mathbf{v}_{m} \in \mathbb{Z}^{d}$ are the outer normals of the facets of the rational polytope $P_{1}+\ldots+P_{n}$. Similarly define the positive halfspace $H_{j}(\mathbf{z})^{+}$and positive open halfspace $H_{j}(\mathbf{z})^{+\circ}$ (as in Notations 22 (1)). Now, given any $I \in \tilde{C}_{k}$, there exists a unique cell $S_{I} \in \tilde{C}_{n}$ such that $I \subseteq \bar{S}_{I}$, the closure of $S_{I}$ in $\mathbb{R}_{>0}^{d}$, and

$$
S_{I} \subseteq H_{j_{1}}\left(\mathbf{z}_{1}\right)^{+o} \cap H_{j_{2}}\left(\mathbf{z}_{2}\right)^{+o} \cap \cdots \cap H_{j_{n-k}}\left(\mathbf{z}_{n-k}\right)^{+o},
$$

where $I \subseteq H_{j_{1}}\left(\mathbf{z}_{1}\right) \cap H_{j_{2}}\left(\mathbf{z}_{2}\right) \cap \cdots \cap H_{j_{n-k}}\left(\mathbf{z}_{n-k}\right)$. Now one can check that $Q\left(P_{1}, \ldots, P_{n}, \mathbf{s}\right)=$ constant for all $\mathbf{s} \in S_{I} \cup I$ and hence the proof follows imitating the rest of the arguments.

Theorem 30 Let $P$ be a convex rational polytope in $\mathbb{R}^{d}=\mathbb{R}^{d-1} \times \mathbb{R}$ of dimension $d$ and let 


$$
i\left(P_{\lambda}, n\right)=\sum_{i=0}^{\operatorname{dim}\left(P_{\lambda}\right)} C_{i}\left(P_{\lambda}, n\right) n^{i}, \text { for } n \in \mathbb{Z}_{\geq 0}
$$

be the Ehrhart quasi-polynomial for $P_{\lambda}:=P \cap\{z=\lambda\} \subset \mathbb{R}^{d-1} \times\{\lambda\}$, for $\lambda \in \mathbb{Q} \geq 0$. Then there are constants, independent of $n, \tilde{c}_{i}(P)$ and $\tilde{c}_{d-1}\left(P_{\lambda}\right)$ such that for $0 \leq i \leq$ $d-1$,

$$
\left|C_{i}\left(P_{\lambda}, n\right)\right| \leq \tilde{c}_{i}(P), \quad \text { and } \quad C_{d-1}\left(P_{\lambda}, n\right)=\tilde{c}_{d-1}\left(P_{\lambda}\right) \text { provided } \lambda n \in \mathbb{Z}_{\geq 0} .
$$

Proof We say a polytope $P$ satisfies $(\star)$ condition if all the vertices of $P$ lie in the union of two hyperplanes $\left\{z=a_{1}\right\} \cup\left\{z=a_{2}\right\}$, for some rational numbers $a_{1}<a_{2}$.

First we prove the theorem for $P$ with the additional $(\star)$ condition.

Let $P_{1}=P \cap\left\{z=a_{1}\right\}$ and $P_{2}=P \cap\left\{z=a_{2}\right\}$. Then

$$
\begin{aligned}
& P_{1}=\text { convex hull }\left\{\left(\mathbf{v}_{1}, a_{1}\right), \ldots,\left(\mathbf{v}_{m}, a_{1}\right)\right\} \text { and } \\
& P_{2}=\text { convex hull }\left\{\left(\mathbf{w}_{1}, a_{2}\right), \ldots,\left(\mathbf{w}_{s}, a_{2}\right)\right\}
\end{aligned}
$$

for a set of some rational points $\left\{\mathbf{v}_{i}, \mathbf{w}_{j}\right\}_{i, j} \subset \mathbb{R}^{d-1}$.

Note that, for $\lambda \in\left[a_{1}, a_{2}\right]$, there is a decomposition as a Minkowski sum

$$
P \cap\{z=\lambda\}=r_{\lambda} P_{1}+r_{\lambda}^{\prime} P_{2}, \quad \text { where } \quad r_{\lambda}=\frac{a_{2}-\lambda}{a_{2}-a_{1}}, \quad r_{\lambda}^{\prime}=\frac{\lambda-a_{1}}{a_{2}-a_{1}} .
$$

Let $\tilde{P}_{1}=$ convex hull of $\left\{\mathbf{v}_{1}, \ldots, \mathbf{v}_{m}\right\}$ and $\tilde{P}_{2}=$ convex hull $\left\{\mathbf{w}_{1}, \ldots, \mathbf{w}_{s}\right\}$. Note that $\operatorname{dim}\left(\tilde{P}_{1}+\tilde{P}_{2}\right)=d-1$, as there exists an isometric map $\tilde{P}_{1}+\tilde{P}_{2} \longrightarrow P_{1}+P_{2}$ given by

$$
\begin{gathered}
\sum_{i} \lambda_{i} \mathbf{v}_{i}+\sum_{j} \mu_{j} \mathbf{w}_{j} \mapsto\left(\sum_{i} \lambda_{i} \mathbf{v}_{i}+\sum_{j} \mu_{j} \mathbf{w}_{j}, a_{1}+a_{2}\right) \\
=\left(\sum_{i} \lambda_{i} \mathbf{v}_{i}, a_{1}\right)+\left(\sum_{j} \mu_{j} \mathbf{w}_{j}, a_{2}\right)
\end{gathered}
$$

and therefore, $\operatorname{dim}\left(P_{1}+P_{2}\right)=\operatorname{dim}\left(2\left(P \cap\left\{z=a_{1}+a_{2} / 2\right\}\right)\right)=d-1$. Hence, by Theorem 28, we have

$$
Q\left(\tilde{P}_{1}, \tilde{P}_{2}, \mathbf{r}\right):=\#\left(\left(r_{1} \tilde{P}_{1}+r_{2} \tilde{P}_{2}\right) \cap \mathbb{Z}^{d-1}\right)=\sum_{l_{1}+l_{2} \leq d-1} p_{l_{1}, l_{2}}(\mathbf{r}) r_{1}^{l_{1}} r_{2}^{l_{2}}, \quad \text { for } \quad \mathbf{r} \in \mathbb{Q}_{\geq 0}^{2},
$$

where, there exist constants $C_{l_{1}, l_{2}}$ and $C_{l_{1}}$ such that

$$
\left|p_{l_{1}, l_{2}}(\mathbf{r})\right| \leq C_{l_{1}, l_{2}} \text { and } \quad p_{l_{1}, d-1-l_{1}}(\mathbf{r})=C_{l_{1}} \quad \text { for all } \quad \mathbf{r} \in \mathbb{Q}_{>0}^{2} .
$$


If $\lambda n \in \mathbb{Z}_{>0}$, then one can check that

$$
i(P \cap\{z=\lambda\}, n)=\#\left(\left(r_{\lambda} n P_{1}+r_{\lambda}^{\prime} n P_{2}\right) \cap \mathbb{Z}^{d}\right)=\#\left(\left(r_{\lambda} n \tilde{P}_{1}+r_{\lambda}^{\prime} n \tilde{P}_{2}\right) \cap \mathbb{Z}^{d-1}\right)
$$

Therefore, by (14),

$$
i(P \cap\{z=\lambda\}, n)=\sum_{l_{1}+l_{2} \leq d-1} p_{l_{1}, l_{2}}\left(r_{\lambda} n, r_{\lambda}^{\prime} n\right) r_{\lambda}^{l_{1}} r_{\lambda}^{\prime l_{2}} n^{l_{1}+l_{2}}=\sum_{i=0}^{d-1} C_{i}\left(P_{\lambda}, n\right) n^{i}
$$

where

$$
C_{i}\left(P_{\lambda}, n\right)=\sum_{l_{1}+l_{2}=i} p_{l_{1}, l_{2}}\left(r_{\lambda} n, r^{\prime}{ }_{\lambda} n\right) r_{\lambda}^{l_{1}} r_{\lambda}^{\prime l_{2}}
$$

Now $a_{1}<\lambda<a_{2}$ and $n \in \mathbb{Z}_{>0}$ implies $\left(r_{\lambda} n, r_{\lambda}^{\prime} n\right) \in \mathbb{Q}_{>0}^{2}$, and therefore, by Theorem 28 and Theorem 20, there exist constants $c_{i}^{\prime}(P)$ and $c_{d-1}^{\prime}\left(P_{\lambda}\right)$ such that $\left|C_{i}\left(P_{\lambda}, n\right)\right| \leq c_{i}^{\prime}(P)$ and $C_{d-1}\left(P_{\lambda}, n\right)=c_{d-1}^{\prime}\left(P_{\lambda}\right)=\mathrm{rVol}_{d-1}\left(P_{\lambda}\right)$ (see Definition 47 for a discussion on the relative volume $\left.\mathrm{rVol}_{d-1}\right)$.

Now $\tilde{c}_{i}(P):=\max \left\{c_{i}^{\prime}(P), C_{i}\left(P_{a_{1}}, n\right), C_{i}\left(P_{a_{2}}, n\right)\right\}$, is finite, by the theory of Ehrhart polynomials for the rational polytopes $P_{a_{1}}$ and $P_{a_{2}}$. Moreover, $C_{d-1}\left(P_{a_{i}}, n\right)$ is constant $\left(=0\right.$, if $\left.\operatorname{dim}\left(P_{a_{i}}\right)<d-1\right)$.

This proves the theorem for a rational polytope $P$ which satisfies the condition $(\star)$.

Consider the projection $\pi: \mathbb{R}^{d} \longrightarrow \mathbb{R}$ to the last coordinate. Let $b_{1}<b_{2}<\cdots<$ $b_{l}$, where $b_{i}$ are the images of the vertices of the polytope $P$. Now $P_{b_{m}}^{b_{m+1}}:=P \cap\left\{b_{m} \leq\right.$ $\left.z \leq b_{m+1}\right\}$ satisfy the condition $(\star)$. Hence the proof of the theorem follows by taking

$$
\tilde{c}_{i}(P)=\max \left\{\tilde{c}_{i}\left(P_{b_{m}}^{b_{m+1}}\right) \mid 1 \leq m \leq l-1\right\} \quad \text { and } \quad \tilde{c}_{d-1}\left(P_{\lambda}\right)=\tilde{c}_{d-1}\left(\left(P_{b_{m}}^{b_{m+1}}\right)_{\lambda}\right),
$$

if $\lambda \in\left[b_{m}, b_{m+1}\right]$.

\section{Main theorem}

We now resume the study of Eto's set $\mathcal{P}_{D}$, and the decompositions $\mathcal{P}_{D}=\cup_{j=1}^{s} P_{j}^{\prime}$ and $\overline{\mathcal{P}}_{D}=\cup_{j=1}^{s} P_{j}$, as discussed in Sect. 3. We will make use of properties of relative volumes, recalled in "Appendix" (see Lemmas 48 and 49).

Notations 31 1. $F(Q)=\{$ the facets of $Q\}$ and $v(Q)=\{$ the vertices of $Q\}$, where $Q$ is a convex polytope.

2. Let $v\left(\mathcal{P}_{D}\right):=\cup_{j=1}^{s} \pi\left(v\left(P_{j}\right)\right)$, where $\pi: \mathbb{R}^{d} \longrightarrow \mathbb{R}$ is the projection given by projecting to the last coordinate $z$ and the set $\pi\left(v\left(P_{j}\right)\right)=\left\{\rho_{j_{1}}, \ldots, \rho_{j_{m}}\right\}$, with $\rho_{j_{1}}<\rho_{j_{2}}<\cdots<\rho_{j_{m_{j}}}$.

3. Let $S=\left\{m / q \mid q=p^{n}, \quad m, n \in \mathbb{Z}_{\geq 0}\right\} \backslash v\left(\mathcal{P}_{D}\right)$. 
Lemma 32 Let $P_{j}$ be a convex polytope as given in Notations 7 (8). Let

$$
i\left(P_{j}, n, \lambda\right)=i\left(P_{j \lambda}, n\right)=\sum_{i=0}^{\operatorname{dim}\left(P_{j \lambda}\right)} C_{i}\left(P_{j \lambda}, n\right) n^{i} \text { for } n \in \mathbb{Z}_{\geq 0}
$$

be the Ehrhart quasi-polynomial for $P_{j \lambda}=P_{j} \cap\{z=\lambda\}$, for $\lambda \in \mathbb{R}_{\geq 0}$. Then for $\lambda \in S$ and $q \lambda \in \mathbb{Z}$, we have

1. $\sum_{j=1}^{s} C_{d-1}\left(P_{j \lambda}, q\right)=f_{R, \mathbf{m}}(\lambda)$.

2. $C_{d-2}\left(P_{j \lambda}, q\right)=\frac{1}{2} \sum_{\tilde{F} \in F\left(P_{j}\right)} \mathrm{rVol}_{d-2}(\tilde{F} \cap\{z=\lambda\})$.

3. For every $i \leq d-3$, we have $\left|C_{i}\left(P_{j \lambda}, q\right)\right| \leq \tilde{c}_{i}\left(P_{j}\right)$ for some constants $\tilde{c}_{i}\left(P_{j}\right)$ independent of $\lambda \in S$.

Proof Let $\lambda=m_{0} / q_{0}$ and $q \geq q_{0}$. Then $q \lambda \in \mathbb{Z}$.

(1) By Theorem 1.1 of [13], for any $\lambda \in \mathbb{R}$, we have $f_{R, \mathbf{m}}(\lambda)=\operatorname{Vol}_{d-1}\left(\mathcal{P}_{D} \cap\{z=\lambda\}\right)$. Also, by the proof of Theorem 1.1 of [13] (see the proof of the claim there), we have

$$
\begin{gathered}
C_{d-1}\left(P_{j \lambda}, q\right)=C_{d-1}\left(P_{j \lambda}\right)=\operatorname{rVol}_{d-1}\left(P_{j \lambda}\right) \\
\text { and } \sum_{j} \operatorname{rVol}_{d-1}\left(P_{j \lambda}\right)=\operatorname{Vol}_{d-1}\left(\mathcal{P}_{D} \cap\{z=\lambda\}\right) .
\end{gathered}
$$

(2) Case (a): If $\lambda \in\left(\rho_{j_{1}}, \rho_{j_{m_{j}}}\right)$, where $\left\{\rho_{j_{1}}, \ldots, \rho_{j_{m_{j}}}\right\}=\pi\left(v\left(P_{j}\right)\right)$ (as in Notations 31), then $\operatorname{dim} P_{j \lambda}=d-1$. Note that the set of facets of $P_{j \lambda}$

$$
F\left(P_{j \lambda}\right)=\left\{\tilde{F} \cap\{z=\lambda\} \mid \tilde{F} \in F\left(P_{j}\right), \operatorname{dim} \tilde{F} \cap\{z=\lambda\}=d-2\right\} .
$$

By Lemma 14 (3), for any $F \in F\left(P_{j \lambda}\right)$, we have $A\left(q_{0} F \cap\left\{z=m_{0}\right\}\right) \cap \mathbb{Z}^{d} \neq \phi$. Hence, for all $q \geq q_{0}$, Theorem 16 implies

$$
C_{d-2}\left(q_{0} P_{j \lambda}, \frac{q}{q_{0}}\right)=\sum_{F \in F\left(P_{j \lambda}\right)} \frac{\mathrm{rVol}_{d-2}\left(q_{0} F_{\lambda}\right)}{2}=\frac{q_{0}^{d-2}}{2} \sum_{F \in F\left(P_{j \lambda}\right)} \operatorname{rVol}_{d-2}\left(F_{\lambda}\right) .
$$

Moreover $\operatorname{rVol}_{d-2}(\tilde{F} \cap\{z=\lambda\})=0$ if $\operatorname{dim}(\tilde{F} \cap\{z=\lambda\})<d-2$.

(2) Case (b): For $\lambda \notin\left[\rho_{j_{1}}, \rho_{j_{m_{j}}}\right]$ we know $i\left(P_{j \lambda}, q\right)=0$, for all $q$.

(3) follows by Theorem 30. This proves the lemma.

Lemma 33 Let $Q_{\alpha}=P_{i} \cap P_{j}$ or $E_{j v}$ (as in Notations 7 and Remark 13), where $P_{i} \neq P_{j}$. Let $\left.i\left(Q_{\alpha \lambda}, n\right)\right)=\sum_{i=0}^{d-2} C_{i}\left(Q_{\alpha \lambda}, n\right) n^{i}$ be the Ehrhart quasi-polynomial for $Q_{\alpha \lambda}=Q_{\alpha} \cap\{z=\lambda\}$ where $\lambda \in \mathbb{Q}_{\geq 0}$. Then, for $\lambda \in S$ and $q \lambda \in \mathbb{Z}_{\geq 0}$, we have

1. $C_{d-2}\left(Q_{\alpha \lambda}, q\right)=\mathrm{rVol}_{d-2}\left(Q_{\alpha \lambda}\right)$. Moreover

2. (a) if $\operatorname{dim}\left(Q_{\alpha}\right)=d-1$, then $\left|C_{i}\left(Q_{\alpha \lambda}, q\right)\right| \leq \tilde{c}_{i}\left(Q_{\alpha}\right)$, for every $i \leq d-3$, and

(b) if $\operatorname{dim}\left(Q_{\alpha}\right) \leq d-2$, then $i\left(Q_{\alpha \lambda}, q\right) \leq C_{\alpha} q^{d-3}$.

Proof Let $\lambda=m_{0} / q_{0}$ and let $q \geq q_{0}$. 
(1) Case (a): If $\operatorname{dim}\left(Q_{\alpha \lambda}\right)=d-2$, then by Lemma 14 (3), for $q \lambda \in \mathbb{Z}_{\geq 0}, A\left(q_{0} Q_{\alpha \lambda}\right) \cap$ $\mathbb{Z}^{d}=A\left(q_{0} Q_{\alpha} \cap\left\{z=m_{0}\right\}\right) \cap \mathbb{Z}^{d} \neq \phi$. Hence, by Theorem 16

$$
C_{d-2}\left(q_{0} Q_{\alpha \lambda}, q / q_{0}\right)=C_{d-2}\left(q_{0} Q_{\alpha \lambda}\right)=\operatorname{rVol}_{d-2}\left(q_{0} Q_{\alpha \lambda}\right)=q_{0}^{d-2} \operatorname{rVol}_{d-2}\left(Q_{\alpha \lambda}\right) .
$$

(1) Case (b): Let $\operatorname{dim}\left(Q_{\alpha \lambda}\right)<d-2$. By Lemma 49, for a convex rational polytope $Q_{\alpha}$, there exists a constant $C_{\alpha}$ such that $i\left(Q_{\alpha \lambda}, q\right) \leq C_{\alpha} q^{\operatorname{dim}\left(Q_{\alpha \lambda}\right)}$. Therefore $C_{d-2}\left(Q_{\alpha \lambda}, q\right)=0=\mathrm{rVol}_{d-2}\left(Q_{\alpha \lambda}\right)$. This proves the first assertion.

(2) if $\operatorname{dim}\left(Q_{\alpha}\right)=d-1$, then by Lemma 48 , there exists a map $\varphi_{\alpha}: \mathbb{R}^{d} \longrightarrow \mathbb{R}^{d-1}$ and $z_{\alpha} \in \mathbb{Z}^{d-1}$ such that $i\left(Q_{\alpha \lambda}, q\right)=i\left(\varphi_{\alpha}\left(Q_{\alpha}\right)_{\lambda}, q\right)$, where $\varphi_{\alpha}\left(Q_{\alpha}\right)$ is a $d-1$ dimensional polytope in $\mathbb{R}^{d-1}$. Hence Assertion 2 (a) follows from Theorem 30. If $\operatorname{dim}\left(Q_{\alpha}\right) \leq d-2$, then $Q_{\alpha}=P_{i} \cap P_{j}$, as $\operatorname{dim} E_{j v}=d-1$ if $E_{j v} \neq \phi$. Without loss of generality we can assume that $\operatorname{dim}\left(Q_{\alpha}\right) \geq 1$. Since $Q_{\alpha}$ is transversal to the hyperplane $\{z=0\}$, we have $\operatorname{dim}\left(Q_{\alpha \lambda}\right)<\operatorname{dim}\left(Q_{\alpha}\right)$, for all $\lambda$. Hence, by Lemma 49 , we have Assertion (2) (b). This completes the proof of the lemma.

Lemma 34 For $Q_{\alpha}^{\prime}$, where $\alpha \in I_{1}$, we have $i\left(Q_{\alpha \lambda}^{\prime}, q\right)=\tilde{c}_{\alpha}^{\prime}(\lambda) q^{d-3}$, where $\left|\tilde{c}_{\alpha}^{\prime}(\lambda)\right|<$ $\tilde{c}_{\alpha}$, for all $\lambda \in \mathbb{Q}_{\geq 0}$.

Proof Note that $\operatorname{dim}\left(Q_{\alpha}^{\prime}\right) \leq d-2$, for $\alpha \in I_{1}$. Hence, by Lemma 14(1), if $\operatorname{dim}\left(Q_{\alpha \lambda}^{\prime}\right)=$ $d-2$, for some $\lambda \in \mathbb{R}_{\geq 0}$, then $\lambda \in v\left(\mathcal{P}_{D}\right)$. Therefore $\lambda \in S \operatorname{implies} \operatorname{dim}\left(Q_{\alpha \lambda}^{\prime}\right) \leq d-3$. Hence the proof follows by Lemma 49.

Definition 35 For a pair $(R, \mathbf{m})$, where $R$ is a standard graded ring of dimension $d$, we define

(1) a sequence of functions $g_{n}:[0, \infty) \longrightarrow \mathbb{R}$, given by

$$
g_{n}(\lambda)=g_{n}(\lfloor\lambda q\rfloor / q)=\frac{1}{q^{d-2}}\left(\ell\left(R / \mathbf{m}^{[q]}\right)\lfloor\lambda q\rfloor-\bar{f}_{n}(\lambda) q^{d-1}\right),
$$

where $f_{R, \mathbf{m}}$ is the HK density function for $(R, \mathbf{m})$ (see Theorem 5 ) and $\bar{f}_{n}(\lambda):=$ $f_{R, \mathbf{m}}\left(\frac{\lfloor\lambda q\rfloor}{q}\right)$.

(2) We also define the $\beta$ density function $g_{R, \mathbf{m}}:[0, \infty) \longrightarrow \mathbb{R}$, given by

$$
\begin{aligned}
g_{R, \mathbf{m}}(\lambda)= & \sum_{\left\{F \in F\left(P_{j}\right), F \subseteq \partial\left(C_{D}\right)\right\}_{j}} \frac{\mathrm{rVol}_{d-2}\left(F_{\lambda}\right)}{2} \\
& -\sum_{\left\{F \in F\left(P_{j}\right), F \subseteq F^{\prime} \in F\left(C_{u}\right), u \in L\left(P_{D}\right)\right\}_{j}} \frac{\mathrm{rVol}_{d-2}\left(F_{\lambda}\right)}{2},
\end{aligned}
$$

where $P_{j}$ and $v\left(\mathcal{P}_{D}\right)$ are as in Notations 7 and in Notations 31 and $F_{\lambda}=F \cap\{z=\lambda\}$. Hence, by Lemma 11 (2),

$$
g_{R, \mathbf{m}}(\lambda)=\operatorname{rVol}_{d-2}\left(\partial\left(\mathcal{P}_{D}\right) \cap \partial\left(C_{D}\right) \cap\{z=\lambda\}\right)-\frac{\operatorname{rVol}_{d-2}\left(\partial\left(\mathcal{P}_{D}\right) \cap\{z=\lambda\}\right)}{2} .
$$


Remark 36 By Lemma 48, for a facet $F$ of $P_{j}$, there exists an invertible affine transformation $\varphi_{F}: A(F) \longrightarrow \mathbb{R}^{d-1}$ and $e_{F}=\varphi\left(z_{1}\right) \in \varphi(A(F)) \cap \mathbb{Z}^{d-1}$ (let $z_{F}$ be the coordinate corresponding to the basis vector $e_{F}$, see Lemma 48) such that for $\lambda \in S=\left\{m / p^{n} \mid m, n \in \mathbb{Z}_{\geq 0}\right\}$,

$$
\operatorname{rVol}_{d-2}(F \cap\{z=\lambda\})=\operatorname{Vol}_{d-2}\left(\varphi_{F}(F) \cap\left\{z_{F}=\lambda\right\}\right) .
$$

Note that $\cup_{j}\left\{v(F) \mid F \in F\left(P_{j}\right)\right\} \subseteq v\left(\mathcal{P}_{D}\right)$. Therefore, by Theorem 2.3 of [13], the function $\psi_{F}:[0, \infty) \backslash v\left(\mathcal{P}_{D}\right) \longrightarrow[0, \infty)$ given by $\lambda \rightarrow \operatorname{Vol}_{d-2}\left(\varphi_{F}(F) \cap\left\{z_{F}=\lambda\right\}\right)$ is continuous.

Thus the function $g_{R, \mathbf{m}}$ is a compactly supported function and is continuous outside the finite set $v\left(\mathcal{P}_{D}\right)$. Moreover, by Lemma 3.4 of [13], $g_{R, \mathbf{m}}$ is a piecewise polynomial function.

Lemma 37 Let $(X, D)$ (as in Notations 4 ) be a toric pair of dimension $d-1 \geq 1$ and let $(R, \mathbf{m})$ be the associated coordinate ring. If $\lambda \in \mathbb{R}_{\geq 0}$ and $q=p^{n} \in \mathbb{N}$ are such that $\lambda_{n}:=\lfloor\lambda q\rfloor / q \in S$, then there exists a constant $\tilde{C}_{\mathcal{P}_{D}}$ such that

$$
g_{n}(\lambda)=g_{R, \mathbf{m}}\left(\lambda_{n}\right)+\tilde{c}\left(\lambda_{n}\right) / q \text {, where }\left|\tilde{c}\left(\lambda_{n}\right)\right| \leq \tilde{C}_{\mathcal{P}_{D}} .
$$

Proof For a polytope $P$, we have $i\left(P, q, \lambda_{n} q\right)=i\left(P_{\lambda_{n}}, q\right)$ and $\ell\left(R / \mathbf{m}^{[q]}\right) \lambda_{n} q=$ $i\left(\left(\mathcal{P}_{D}\right)_{\lambda_{n}}, q\right)$, where, by Remark 13 (6), we have

$$
\begin{aligned}
i\left(\left(\mathcal{P}_{D}\right)_{\lambda_{n}}, q\right)= & \sum_{j} i\left(\left(P_{j}\right)_{\lambda_{n}}, q\right)-\sum_{j<k} i\left(\left(P_{j} \cap P_{k}\right)_{\lambda_{n}}, q\right) \\
& -\sum_{j, \gamma} i\left(\left(E_{j \gamma}\right)_{\lambda_{n}}, q\right)+\sum_{\alpha \in I_{1}} \epsilon_{\alpha} i\left(\left(Q_{\alpha}^{\prime}\right)_{\lambda_{n}}, q\right) .
\end{aligned}
$$

Now, by Lemma 32, for $1 \leq j \leq s$, we have

$$
\begin{gathered}
i\left(\left(P_{j}\right)_{\lambda_{n}}, q\right)-\mathrm{rVol}_{d-1}\left(\left(P_{j}\right) \lambda_{n}\right) q^{d-1}=\frac{1}{2} \sum_{\left\{F \in F\left(P_{j}\right)\right\}} \mathrm{rVol}_{d-2}\left(F_{\lambda_{n}}\right) q^{d-2}+\tilde{c}_{j}\left(\lambda_{n}\right) q^{d-3} \\
=\left[\sum_{\left\{F \mid F=P_{i} \cap P_{j}\right\}_{i}} \frac{\mathrm{rVol}_{d-2}\left(F_{\lambda_{n}}\right)}{2}+\sum_{\substack{\left\{F \mid F \subseteq \partial\left(C_{D}\right)\right\} \\
+\tilde{c}_{j}\left(\lambda_{n}\right) q^{d-3},}} \frac{\mathrm{rVol}_{d-2}\left(F_{\lambda_{n}}\right)}{2}+\sum_{\{\nu\}} \frac{\mathrm{rVol}_{d-2}\left(E_{j v}\right) \lambda_{n}}{2}\right] q^{d-2}
\end{gathered}
$$

where $F \in F\left(P_{j}\right)$ and $\left|\tilde{c}_{j}\left(\lambda_{n}\right)\right| \leq \tilde{c}_{j}$, for some constant $c_{j}$ independent of $\lambda_{n}$.

Hence, by Lemma 32,

$$
\begin{gathered}
\sum_{j} i\left(\left(P_{j}\right) \lambda_{n}, q\right)-f_{R, \mathbf{m}}\left(\lambda_{n}\right) q^{d-1} \\
=\left[\sum_{\left\{F=P_{i} \cap P_{j} \mid i<j\right\}_{i, j}} \operatorname{rVol}_{d-2}\left(F_{\lambda_{n}}\right)+\sum_{\left\{F \mid F \subseteq \partial\left(C_{D}\right)\right\}_{j}} \frac{\mathrm{rVol}_{d-2}\left(F_{\lambda_{n}}\right)}{2}+\sum_{\{j, v\}} \frac{\mathrm{rVol}_{d-2}\left(E_{j v}\right) \lambda_{n}}{2}\right] q^{d-2} \\
+\sum_{j} \tilde{c}_{j}\left(\lambda_{n}\right) q^{d-3}
\end{gathered}
$$


and by Lemma 33,

$$
\sum_{i<j} i\left(\left(P_{i} \cap P_{j}\right)_{\lambda_{n}}, q\right)=\sum_{\left\{F=P_{i} \cap P_{j}\right\}_{i<j}} \operatorname{rVol}_{d-2}\left(F_{\lambda_{n}}\right) q^{d-2}+\tilde{c}_{i j}\left(\lambda_{n}\right) q^{d-3},
$$

where $\left|\tilde{c}_{i j}\left(\lambda_{n}\right)\right| \leq \tilde{c}_{i j}$.

Now, by Lemmas 33 and 34, we have

$$
\begin{aligned}
\frac{1}{q^{d-2}} \ell\left(\frac{R}{\mathbf{m}^{[q]}}\right)_{\lambda_{n} q}-f_{R, \mathbf{m}}\left(\lambda_{n}\right) q= & \sum_{\left\{F \in F\left(P_{j}\right), F \subseteq \partial\left(C_{D}\right)\right\}_{j}} \frac{\mathrm{rVol}_{d-2}\left(F_{\lambda_{n}}\right)}{2} \\
& -\sum_{\{j, \nu\}} \frac{\mathrm{rVol}_{d-2}\left(E_{j \nu}\right) \lambda_{n}}{2}+\frac{\tilde{c}\left(\lambda_{n}\right)}{q},
\end{aligned}
$$

where $\left|\tilde{c}\left(\lambda_{n}\right)\right| \leq \tilde{C}_{\mathcal{P}_{D}}$. Hence $g_{n}(\lambda)=g_{R, \mathbf{m}}\left(\lambda_{n}\right)+\tilde{c}\left(\lambda_{n}\right) / q$, where $\left|\tilde{c}\left(\lambda_{n}\right)\right| \leq \tilde{C}_{\mathcal{P}_{D}}$. This implies the lemma.

Remark 38 By construction, it follows that $\operatorname{Support}\left(g_{R, \mathbf{m}}\right) \cup_{n} \operatorname{Support}\left(g_{n}\right) \subseteq \pi\left(\overline{\mathcal{P}}_{D}\right)$, which is a compact set and where $\pi: \mathbb{R}^{d} \rightarrow \mathbb{R}$ is the projection as in Notations 31 (2).

Lemma 39 The function $g_{R, \mathbf{m}}$ is a compactly supported function and is continuous on $[0, \infty) \backslash v\left(\mathcal{P}_{D}\right)$.

1. For any given compact set $V \subseteq[0, \infty) \backslash v\left(\mathcal{P}_{D}\right)$, the sequence $\left.g_{n}\right|_{V}$ converges uniformly to $g_{R, \mathbf{m}} \mid V$.

2. $\int_{0}^{\infty} g_{n}(\lambda) \mathrm{d} \lambda=\int_{0}^{\infty} g_{R, \mathbf{m}}(\lambda) \mathrm{d} \lambda+O(1 / q)$.

Proof By Remark 36, the function $g_{R, \mathbf{m}}$ is a compactly supported function and is continuous outside $v\left(\mathcal{P}_{D}\right)$.

(1) Let us fix a compact set $V \subseteq[0, \infty) \backslash v\left(\mathcal{P}_{D}\right)$. We can assume $V \subseteq\left[0, \pi\left(\overline{\mathcal{P}}_{D}\right)\right] \backslash v$ $\left(P_{D}\right)$. Now, let $\left[0, \pi\left(\overline{\mathcal{P}}_{D}\right)\right] \backslash v\left(\mathcal{P}_{D}\right)=\cup_{i=1}^{m}\left(b_{i}, b_{i+1}\right)$, where $\left.g_{R, \mathbf{m}}\right|_{\left(b_{i}, b_{i+1}\right)}=\Psi_{i}$, for some polynomial function $\Psi_{i}$. We choose $q_{0}=p^{n_{0}}$ such that

$$
V \subseteq \cup_{i=1}^{m}\left[b_{i}+2 / q_{0}, b_{i+1}-2 / q_{0}\right] \subseteq \cup_{i=1}^{m}\left[b_{i}, b_{i+1}\right]
$$

Now, $q \geq q_{0}$ and $\lambda \in V \cap\left(b_{i}, b_{i+1}\right)$ imply $\lambda_{n} \in\left[b_{i}+1 / q_{0}, b_{i+1}-1 / q_{0}\right]$. Hence

$$
\left|g_{R, \mathbf{m}}(\lambda)-g_{R, \mathbf{m}}\left(\lambda_{n}\right)\right|=\left|\Psi_{i}(\lambda)-\Psi_{i}\left(\lambda_{n}\right)\right| \leq C_{i} / q,
$$

where $C_{i}$ is a constant determined by $P_{i}$ and is independent of $q$. By Lemma 37, we have $\left|g_{n}(\lambda)-g_{R, \mathbf{m}}(\lambda)\right| \leq C_{i} / q+\tilde{C}_{\mathcal{P}_{D}} / q$, for all $\lambda \in V \cap\left(b_{i}, b_{i+1}\right)$ and $q \geq q_{0}$. Hence $\left|g_{n}(\lambda)-g_{R, \mathbf{m}}(\lambda)\right| \leq c_{0} / q$, for alll $\lambda \in V$ and $q \geq q_{0}$. This proves part (1) of the lemma.

(2) Fix $q_{0}=p^{n_{0}}$ such that $\cup_{i=1}^{m}\left[b_{i}+2 / q_{0}, b_{i+1}-2 / q_{0}\right] \subseteq \cup_{i=1}^{m}\left[b_{i}, b_{i+1}\right]$. For $q=$ $p^{n}$, if $q \geq q_{0}$, then $V_{1}:=\cup_{i=1}^{m}\left[b_{i}+2 / q, b_{i+1}-2 / q\right] \subseteq \cup_{i=1}^{m}\left[b_{i}, b_{i+1}\right]$. Now, arguing as above one can deduce that there is a constant $c_{0}$ such that $\left|g_{n}(\lambda)-g_{R, \mathbf{m}}(\lambda)\right| \leq c_{0} / q$, for alll $\lambda \in V_{1}$. Moreover $\mu\left(\left[0, \pi\left(\overline{\mathcal{P}}_{D}\right) \backslash V_{1}\right) \leq 4 m / q\right.$ (here $\mu$ denotes the Lebesgue 
measure in $\mathbb{R}^{1}$ ). Note that all the functions $g_{n}$ and $g$ are bounded with support in $\left[0, \pi\left(\overline{\mathcal{P}}_{D}\right)\right]$. Hence

$$
\int_{0}^{\infty}\left|g_{n}(\lambda)-g(\lambda)\right| \mathrm{d} \lambda \leq \int_{V_{1}}\left|g_{n}(\lambda)-g(\lambda)\right| \mathrm{d} \lambda+\int_{[0, \infty) \backslash V_{1}}\left|g_{n}(\lambda)-g(\lambda)\right| \mathrm{d} \lambda=O(1 / q) .
$$

The same assertion follows for $q<q_{0}$, by the boundedness of $g$ and $g_{n}$. This proves the part (2) of the lemma, and hence the lemma.

Lemma 40 Let $f:[0, \infty) \rightarrow[0, \infty)$ be a continuous compactly supported piecewise polynomial function. For $q=p^{n}$, let $\tilde{f}_{n}:[0, \infty) \longrightarrow[0, \infty)$ be the function given by $\tilde{f}_{n}(x)=f(\lfloor q x\rfloor / q)$. Then, for all $q=p^{n}$, we have

$$
\int_{0}^{\infty} \tilde{f}_{n}(x) \mathrm{d} x=\int_{0}^{\infty} f(x) \mathrm{d} x+O\left(1 / q^{2}\right) .
$$

Proof We assume the following claim for the moment.

Claim If $P(x) \in \mathbb{R}[x]$ is a polynomial function and $\tilde{P}_{n}(x):=P(\lfloor q x\rfloor / q)$, then

$$
\int_{0}^{A}\left[P(x)-\tilde{P}_{n}(x)\right] \mathrm{d} x=P(A) / 2 q+O\left(1 / q^{2}\right) .
$$

Now, since $f$ is a compactly supported piecewise polynomial continuous function, there exist $0=b_{0}<b_{1}<\ldots<b_{v+1}$ and polynomials $\Phi_{0}(x), \ldots, \Phi_{v}(x) \in \mathbb{R}[x]$ such that $\left.f\right|_{\left[b_{i}, b_{i+1}\right]}=\Phi_{i}(x)$ and $\operatorname{Support}(f) \subseteq\left[b_{0}, b_{\nu+1}\right]$ and $f\left(b_{0}\right)=f\left(b_{v+1}\right)=0$.

Now

$$
\begin{gathered}
\int_{0}^{\infty} f(x) \mathrm{d} x-\int_{0}^{\infty} \tilde{f}_{n}(x) \mathrm{d} x=\int_{0}^{\infty}\left(f(x)-\tilde{f}_{n}(x)\right) \mathrm{d} x \\
=\sum_{i=0}^{v} \int_{b_{i}}^{b_{i+1}}\left(\Phi_{i}(x)-\left(\tilde{\Phi}_{i}\right)_{n}(x)\right) \mathrm{d} x-\int_{b_{i}}^{b_{i}+\frac{1}{q}}\left(\tilde{f}_{n}(x)-\left(\tilde{\Phi}_{i}\right)_{n}(x)\right) \mathrm{d} x \\
=\sum_{i=0}^{v}\left[\int_{0}^{b_{i+1}}\left(\Phi_{i}(x)-\left(\tilde{\Phi}_{i}\right)_{n}(x)\right) \mathrm{d} x-\int_{0}^{b_{i}}\left(\Phi_{i}(x)-\left(\tilde{\Phi}_{i}\right)_{n}(x)\right) \mathrm{d} x\right]+O\left(\frac{1}{q^{2}}\right),
\end{gathered}
$$

where $\left(\tilde{\Phi}_{i}\right)_{n}(x)=\Phi_{i}(\lfloor q x\rfloor / q)$. Therefore, by the above claim

$$
\begin{aligned}
\int_{0}^{\infty}\left[f(x)-\tilde{f}_{n}(x)\right] \mathrm{d} x & =\sum_{i=0}^{v}\left[\frac{\Phi_{i}\left(b_{i+1}\right)}{2 q}-\frac{\Phi_{i}\left(b_{i}\right)}{2 q}\right]+O\left(\frac{1}{q^{2}}\right) \\
& =\sum_{i=0}^{v}\left[\frac{f\left(b_{i+1}\right)}{2 q}-\frac{f\left(b_{i}\right)}{2 q}\right]+O\left(\frac{1}{q^{2}}\right)=O\left(\frac{1}{q^{2}}\right) .
\end{aligned}
$$

Proof of the Claim We can assume without loss of generality that $P(x)=x^{l}$, for some $l \geq 1$. Note that there is $q_{0}=p^{n_{0}}$ such that, for any $q \geq q_{0}$, there is $l_{0}$ such that $l_{0} / q \leq A<\left(l_{0}+1\right) / q$. Then

$$
\int_{0}^{A}\left(P(x)-\tilde{P}_{n}(x)\right) \mathrm{d} x=\int_{0}^{l_{0} / q}\left(P(x)-\tilde{P}_{n}(x)\right) \mathrm{d} x+O\left(1 / q^{2}\right),
$$


as the inequalities $\left(A-l_{0} / q\right) \leq 1 / q$ and $l \geq 1$ implies $\left|\int_{l_{0} / q}^{A}\left(P(x)-\tilde{P}_{n}(x)\right) \mathrm{d} x\right|=$ $O\left(1 / q^{2}\right)$. It is also obvious that $P(A) / 2 q+O\left(1 / q^{2}\right)=P\left(l_{0} / q\right) / 2 q+O\left(1 / q^{2}\right)$.

Now, if $m \in \mathbb{Z}_{\geq 0}$ then

$$
\begin{aligned}
& \int_{\frac{m}{q}}^{\frac{m+1}{q}}\left(P(x)-\tilde{P}_{n}(x)\right) \mathrm{d} x=\int_{\frac{m}{q}}^{\frac{m+1}{q}}\left(x^{l}-(m / q)^{l}\right) \mathrm{d} x \\
& =\int_{\frac{m}{q}}^{\frac{m+1}{q}}\left[\sum_{i=0}^{l-1}\left(\begin{array}{l}
l \\
i
\end{array}\right)(m / q)^{i}(x-m / q)^{l-i}\right] \mathrm{d} x \\
& =\int_{0}^{1 / q}\left[\sum_{i=0}^{l-1}\left(\begin{array}{l}
l \\
i
\end{array}\right)(m / q)^{i}(t)^{l-i}\right] d t=\sum_{i=0}^{l-1}\left(\begin{array}{l}
l \\
i
\end{array}\right)(m / q)^{i} \frac{1}{(l-i+1)\left(q^{l-i+1}\right)}
\end{aligned}
$$

Now $\sum_{m=0}^{q A} m^{i} / q^{l+1}=A^{i+1} / q^{l-i}(i+1)+O\left(1 / q^{l-i+1}\right)$. This implies

$$
\begin{aligned}
& \int_{0}^{A}\left(P(x)-\tilde{P}_{n}(x)\right) \mathrm{d} x=\sum_{i=0}^{q A-1} \int_{m / q}^{m+1 / q}\left(P(x)-\tilde{P}_{n}(x)\right) \mathrm{d} x \\
& =\sum_{i=0}^{l-1}\left[\left(\begin{array}{l}
l \\
i
\end{array}\right) \frac{1}{(l-i+1)(i+1)} \frac{A^{i+1}}{q^{l-i}}+O\left(1 / q^{l-i+1}\right)\right] \\
& =\frac{A^{l}}{2 q}+\cdots+\frac{A^{2}}{2 q^{l-1}}+\frac{A}{(l+1) q^{l}}+O\left(1 / q^{2}\right)=P(A) / 2 q+O\left(1 / q^{2}\right) .
\end{aligned}
$$

This proves the claim and hence the lemma.

Proof (Proof of the Main Theorem) Now the proof follows by putting together Definition 35 (16), Lemma 39 and taking $f=f_{R, \mathbf{m}}$ in Lemma 40.

Proof (Proof of the Corollary 3) Let $g_{R, \mathbf{m}}$ and $g_{n}$ denote the function as given in Definition 35.

The formula for the integral of $g_{R, \mathbf{m}}$ follows from the definition of $g_{R, \mathbf{m}}$ and Fubini's theorem.

For $q=p^{n}$ and for the function $\tilde{f}_{n}$ given by $\tilde{f}_{n}(x)=f_{R, \mathbf{m}}(\lfloor q x\rfloor / q)$, we have

$$
\begin{aligned}
\int_{0}^{\infty} g_{n}(\lambda) \mathrm{d} \lambda & =\frac{1}{q^{d-2}} \int_{0}^{\infty}\left(\ell\left(\left(R / \mathbf{m}^{[q]}\right)_{\lfloor q \lambda\rfloor}\right)-\tilde{f}_{n}(\lambda) q^{d-1}\right) \mathrm{d} \lambda \\
& =\frac{1}{q^{d-2}}\left(\sum_{m=0}^{\infty} \int_{\frac{m}{q}}^{\frac{m+1}{q}} \ell\left(\left(R / \mathbf{m}^{[q]}\right)_{\lfloor q \lambda\rfloor}\right)-q^{d-1} \int \tilde{f}_{n}(\lambda) \mathrm{d} \lambda\right) \\
& =\frac{1}{q^{d-2}}\left(\sum_{m=0}^{\infty} \frac{1}{q} \ell\left(\left(R / \mathbf{m}^{[q]}\right)_{m}\right)-q^{d-1} \int \tilde{f}_{n}(\lambda) \mathrm{d} \lambda\right) .
\end{aligned}
$$


By Lemma 40,

$$
\int_{0}^{\infty} g_{n}(\lambda) \mathrm{d} \lambda=\frac{\ell\left(R / \mathbf{m}^{[q]}\right)}{q^{d-1}}-q \int_{0}^{\infty} f_{R, \mathbf{m}}(\lambda) \mathrm{d} \lambda+O(1 / q) .
$$

hence by Theorem 1.1 of [T2] and part (2) of Lemma 39,

$$
\int_{0}^{\infty} g_{R, \mathbf{m}}(\lambda) \mathrm{d} \lambda+O(1 / q)=\frac{\ell\left(R / \mathbf{m}^{[q]}\right)}{q^{d-1}}-(q) e_{H K}(R, \mathbf{m})+O(1 / q)
$$

which implies

$$
\ell\left(R / \mathbf{m}^{[q]}\right)=e_{H K}(R, \mathbf{m}) q^{d}+q^{d-1} \int_{0}^{\infty} g_{R, \mathbf{m}}(\lambda) \mathrm{d} \lambda+O\left(q^{d-2}\right) .
$$

This gives the corollary.

Remark 41 Since $\partial \mathcal{P}_{D}$ and $\partial \mathcal{P}_{D} \cap \partial C_{D}$ consist of rational $d-1$ dimensional convex polytopes, the number $\beta(R, \mathbf{m})$ (the volume of the integral) is a rational number and also is independent of the characteristic. We note that the above argument gives a direct proof of the result of [9] in our particular situation, for the graded ring $R$. However, the assertion that $\beta(R, \mathbf{m})$ is a constant and rational has been proved earlier by Bruns-Gubeladze in [1], for any normal affine monoid.

It is an interesting problem to extend the computations here to the case of $R$ modules, and to determine the homomorphism $C l(R) \longrightarrow \mathbb{R}$ of [9] in this toric case.

\section{Some properties and examples}

Definition 42 Let $R$ be a Noetherian standard graded ring of dimension $d \geq 2$ with the maximal homogeneous ideal $\mathbf{m}$. Let $\ell\left(R_{n}\right)=\frac{e_{0}(R, \mathbf{m})}{(d-1) !} n^{d-1}+\tilde{e}_{1}(R, \mathbf{m}) n^{d-2}+$ $\cdots+\tilde{e}_{d-1}(R, \mathbf{m})$ be the Hilbert polynomial of $(R, \mathbf{m})$. We define the Hilbert density function $F_{R}:[0, \infty) \longrightarrow[0, \infty)$, of $R$ as

$$
F_{R}(\lambda)=\frac{e_{0}(R, \mathbf{m})}{(d-1) !} \lambda^{d-1}=\lim _{n \rightarrow \infty} F_{n}(\lambda):=\frac{1}{q^{d-1}} \ell\left(R_{\lfloor q \lambda\rfloor}\right) .
$$

Similarly, we can define the second Hilbert density function $G_{R}:[0, \infty) \longrightarrow \mathbb{R}$ as

$$
G_{R}(\lambda)=\tilde{e}_{1}(R, \mathbf{m}) \lambda^{d-2}=\lim _{n \rightarrow \infty} G_{n}(\lambda):=\frac{1}{q^{d-2}}\left(\ell\left(R_{\lfloor q \lambda\rfloor}\right)-F_{R}\left(\frac{\lfloor q \lambda\rfloor}{q}\right)\right) .
$$

Remark 43 Let $R$ and $S$ be two Noetherian standard graded rings over an algebraically closed field $K$ of dimension $d \geq 2$ and $d^{\prime} \geq 2$ with homogenous maximal ideals $\mathbf{m}$ and $\mathbf{n}$, respectively. Then, using the Kunneth formula for sheaf cohomology, it is easy to see

$$
\tilde{e}_{1}(R \# S, \mathbf{m} \# \mathbf{n})=\frac{e_{0}(R, \mathbf{m})}{(d-1) !} \tilde{e}_{1}(R, \mathbf{m})+\frac{e_{0}(S, \mathbf{n})}{\left(d^{\prime}-1\right) !} \tilde{e}_{1}(S, \mathbf{n}) .
$$


Hence we have $G_{R \# S}=G_{R} F_{S}+G_{S} F_{R}$.

Proposition 44 Let $(R, \mathbf{m})$ and $(S, \mathbf{n})$ be two Noetherian standard graded rings over an algebraically closed field $K$ (of characteristic $p>0$ ) of dimension $d \geq 2$ and $d^{\prime} \geq 2$, associated with the toric pairs $(X, D)$ and $\left(Y, D^{\prime}\right)$, respectively. Then we have,

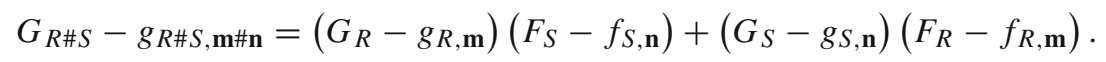

Here the functions $g_{R, \mathbf{m}}$ and $g_{S, \mathbf{n}}$ denote the $\beta$-density functions for the pairs $(R, \mathbf{m})$

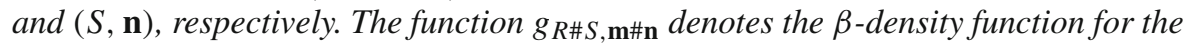
$\operatorname{pair}(R \# S, \mathbf{m \# n})$.

Proof Let $\lambda \in[0, \infty)$. For $n \in N$ and $q=p^{n}$, we write $m=\lfloor q \lambda\rfloor$. Then for the Noetherian standard graded ring $R \# S$ with homogenous maximal ideal $\mathbf{m} \# \mathbf{n}$, we have

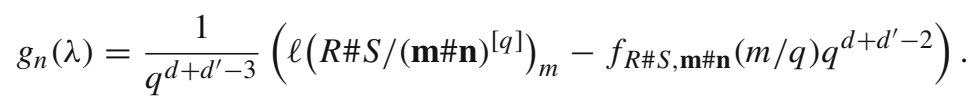

By Proposition 2.17, [18], we have

$$
\begin{aligned}
g_{n}(\lambda)= & \frac{1}{q^{d+d^{\prime}-3}}\left[\ell\left(R_{m}\right) \ell\left(S / \mathbf{n}^{[q]}\right)_{m}+\ell\left(S_{m}\right) \ell\left(R / \mathbf{m}^{[q]}\right)_{m}-\ell\left(R / \mathbf{m}^{[q]}\right)_{m} \ell\left(S / \mathbf{n}^{[q]}\right)_{m}\right] \\
& -\left[F_{R}(m / q) f_{S, \mathbf{n}}(m / q)+F_{S}(m / q) f_{R, \mathbf{m}}(m / q)-f_{R, \mathbf{m}}(m / q) f_{S, \mathbf{n}}(m / q)\right] q . \\
= & \Phi_{n}(\lambda)+\Psi_{n}(\lambda)+\xi_{n}(\lambda),
\end{aligned}
$$

where

$$
\begin{aligned}
& \Phi_{n}(\lambda)=\frac{1}{q^{d+d^{\prime}-3}}\left[\ell\left(R_{m}\right) \ell\left(S / \mathbf{n}^{[q]}\right)_{m}-F_{R}(m / q) f_{S, \mathbf{n}}(m / q) q^{d+d^{\prime}-2}\right] \\
& \Psi_{n}(\lambda)=\frac{1}{q^{d+d^{\prime}-3}}\left[\ell\left(S_{m}\right) \ell\left(R / \mathbf{m}^{[q]}\right)_{m}-F_{S}(m / q) f_{R, \mathbf{m}}(m / q) q^{d+d^{\prime}-2}\right]
\end{aligned}
$$

and

$$
\xi_{n}(\lambda)=-\frac{1}{q^{d+d^{\prime}-3}}\left[\ell\left(R / \mathbf{m}^{[q]}\right)_{m} \ell\left(S / \mathbf{n}^{[q]}\right)_{m}-f_{R, \mathbf{m}}(m / q) f_{S, \mathbf{n}}(m / q) q^{d+d^{\prime}-2}\right] .
$$

We have

$$
\begin{aligned}
\Phi_{n}(\lambda)= & \frac{1}{q^{d+d^{\prime}-3}}\left[\ell\left(R_{m}\right) \ell\left(S / \mathbf{n}^{[q]}\right)_{m}-F_{R}(m / q) f_{S, \mathbf{n}}(m / q) q^{d+d^{\prime}-2}\right] \\
= & \frac{1}{q^{d-1}} \ell\left(R_{m}\right) \times \frac{1}{q^{d^{\prime}-2}}\left[\left(\ell\left(S / \mathbf{n}^{[q]}\right)_{m}-f_{S, \mathbf{n}}(m / q) q^{d^{\prime}-1}\right)\right] \\
& +f_{S, \mathbf{n}}(m / q) \times \frac{1}{q^{d-2}}\left[\ell\left(R_{m}\right)-F_{R}(m / q) q^{d-1}\right] .
\end{aligned}
$$

Thus $\lim _{n \rightarrow \infty} \Phi_{n}(\lambda)=F_{R}(\lambda) g_{S, \mathbf{n}}(\lambda)+f_{S, \mathbf{n}}(\lambda) G_{R}(\lambda)$. Similarly $\lim _{n \rightarrow \infty} \Psi_{n}(\lambda)=$ $F_{S}(\lambda) g_{R, \mathbf{m}}(\lambda)+f_{R, \mathbf{m}}(\lambda) G_{S}(\lambda)$ and $\lim _{n \rightarrow \infty} \xi_{n}(\lambda)=f_{R, \mathbf{m}}(\lambda) g_{S, \mathbf{n}}(\lambda)+f_{S, \mathbf{n}}(\lambda)$ $g_{R, \mathbf{m}}(\lambda)$. This implies, 


$$
g_{R \# S, \mathbf{m} \# \mathbf{n}}=F_{R} g_{S, \mathbf{n}}+f_{S, \mathbf{n}} G_{R}+F_{S} g_{R, \mathbf{m}}+f_{R, \mathbf{m}} G_{S}+f_{R, \mathbf{m}} g_{S, \mathbf{n}}+f_{S, \mathbf{n}} g_{R, \mathbf{m}} .
$$

By Remark 43, we have

$$
G_{R \# S}-g_{R \# S, \mathbf{m} \# \mathbf{n}}=\left(G_{R}-g_{R, \mathbf{m}}\right)\left(F_{S}-f_{S, \mathbf{n}}\right)+\left(G_{S}-g_{S, \mathbf{n}}\right)\left(F_{R}-f_{R, \mathbf{m}}\right) .
$$

Example 45 Consider the toric pair $\left(\mathbb{P}^{2},-K\right)$, where $-K$ is the anticanonical divisor of $\mathbb{P}^{2}$, and let $(R, \mathbf{m})$ be the associated coordinate ring with the homogeneous maximal ideal $\mathbf{m}$. The Hilbert-Kunz function of $(R, \mathbf{m})$ is $\operatorname{HK}(R, \mathbf{m})(q)=q^{3}+O(q)$. For $\lambda \in \mathbb{R}_{\geq 0}$ and for $q=p^{n}$, let $f_{n}(R, \mathbf{m})$ and $f_{R, \mathbf{m}}$ be as in given in Theorem 5 and $g_{n}$ and $g_{R, \mathbf{m}}$ be as in Definition 35. A simple calculation shows

$$
f_{n}(\lambda)= \begin{cases}\frac{1}{2 q^{2}}(m+2)(m+1) & \text { if } 0 \leq \lambda<1, \\ \frac{1}{2 q^{2}}((m+2)(m+1)-3(m-q+2)(m-q+1)) & \text { if } 1 \leq \lambda<2, \\ \frac{1}{2 q^{2}}[(m+2)(m+1)-3(m-q+2)(m-q+1) & \\ +3(m-2 q+2)(m-2 q+1)] & \text { if } 1 \leq \lambda<2 .\end{cases}
$$

Hence

$$
\begin{aligned}
& f_{R, \mathbf{m}}(\lambda)= \begin{cases}\frac{1}{2} \lambda^{2} & \text { if } 0 \leq \lambda<1, \\
\frac{1}{2} \lambda^{2}-\frac{3}{2}(\lambda-1)^{2} & \text { if } 1 \leq \lambda<2, \\
\frac{1}{2} \lambda^{2}-\frac{3}{2}(\lambda-1)^{2}+\frac{3}{2}(\lambda-2)^{2} & \text { if } 1 \leq \lambda<2\end{cases} \\
& g_{n}(\lambda)= \begin{cases}\frac{1}{q}\left(\frac{3}{2} m+1\right) & \text { if } 0 \leq \lambda<1 \\
\frac{1}{q}\left(-3 m+\frac{9}{2} q-2\right) & \text { if } 1 \leq \lambda<2 \\
\frac{1}{q}\left(\frac{3}{2} m-\frac{9}{2} q+1\right) & \text { if } 1 \leq \lambda<2\end{cases} \\
& g_{R, \mathbf{m}}(\lambda)= \begin{cases}\frac{3}{2} \lambda & \text { if } 0 \leq \lambda<1 \\
-3 \lambda+\frac{9}{2} & \text { if } 1 \leq \lambda<2 \\
\frac{3}{2} \lambda-\frac{9}{2} & \text { if } 2 \leq \lambda<3\end{cases}
\end{aligned}
$$

and $\int_{0}^{\infty} g_{R, \mathbf{m}}(\lambda) \mathrm{d} \lambda=0$.

Example 46 We compute the $\beta$-density function for the Hirzebruch surface $X=\mathbb{F}_{a}$ with parameter $a \in \mathbb{N}$, which is a ruled surface over $\mathbb{P}_{K}^{1}$, where $K$ is a field of characteristic $p>0$. See [17] for a detailed description of the surface as a toric variety. The $T$-Cartier divisors are given by $D_{i}=V\left(v_{i}\right), i=1,2,3,4$, where $v_{1}=$ $e_{1}, v_{2}=e_{2}, v_{3}=-e_{1}+a e_{2}, v_{4}=-e_{2}$ and $V\left(v_{i}\right)$ denotes the $T$-orbit closure corresponding to the cone generated by $v_{i}$. We know the Picard group is generated 
by $\left\{D_{i}: i=1,2,3,4\right\}$ over $\mathbb{Z}$. One can check the only relations in $\operatorname{Pic}(X)$ can be described by $D_{3} \sim D_{1}$ and $D_{2} \sim D_{4}-a D_{1}$. Therefore $\operatorname{Pic}(X)=\mathbb{Z} D_{1} \oplus \mathbb{Z} D_{4}$. One can use standard method of toric geometry to see that $D=c D_{1}+d D_{4}$ is ample if and only if $a, c>0$. Then $P_{D}=\left\{(x, y) \in M_{\mathbb{R}} \mid x \geq-c, y \leq d, x \leq a y\right\}$ and $\alpha^{2}=\operatorname{Vol}\left(P_{D}\right)=c d+\frac{a d^{2}}{2}$. For a detailed analysis of Hilbert-Kunz function and Hilbert-Kunz density function of $\mathbb{F}_{a}$, see [17]. In the following we use results from [17] to calculate $\left\{f_{n}(R, \mathbf{m})\right\}$ and $f_{R, \mathbf{m}}$ in order to describe the $\beta$-density function. One can also use the computations from Example 7.2 of [13].

If $c \geq d$, then $f_{R, \mathbf{m}}(q)=(*)$, where

$$
\begin{aligned}
(*)= & q^{3}\left(c+\frac{a d}{2}\right)\left[\frac{d}{3}+\frac{(d+1) d}{6 c(a d+c)}+\frac{1}{2}+\frac{1}{6 d}\right] \\
& +q^{2}\left(\left(c+\frac{a d}{2}\right)(d+1)\left[\frac{1}{4 c}+\frac{1}{4(a d+c)}-\frac{d}{2 c(a d+c)}-\frac{1}{2 d}\right]+\frac{d+1}{2}\right)+O(q)
\end{aligned}
$$

and if $c<d$ then $f_{R, \mathbf{m}}(q)=$

$$
\begin{gathered}
(*)+q^{3} d\left(c+\frac{(d+1) a}{2}\right)\left(\frac{(a+1)^{3}}{6 a(a d+c)}-\frac{1}{6 a c}-\frac{a}{6 d}-\frac{1}{2 d}+\frac{c}{6 d^{2}}\right) \\
-q^{2} d\left(c+\frac{(d+1) a}{2}\right)\left[\frac{(a+2)(a+1)^{3}}{4 a(c+a d)}+\frac{a-2}{4 a c}-\frac{a+2}{4 d}-\frac{1}{d}+\frac{c}{2 d^{2}}\right]+O(q) .
\end{gathered}
$$

An easy but tedious calculation shows that, for $c \geq d$

$$
g_{R, \mathbf{m}}(\lambda)= \begin{cases}\left(c+\frac{a d}{2}+d\right) \lambda & \text { if } 0 \leq \lambda<1 \\ -\left(c+\frac{a d}{2}+d\right)\left(c d+\frac{a d^{2}}{2}+c+\frac{a d}{2}+d\right) \lambda & \\ +\left(c+\frac{a d}{2}+d\right)(d+1)\left(c+\frac{a d}{2}+1\right) & \text { if } 1 \leq \lambda<1+\frac{1}{c+a d} \\ d(d+1)-d\left(c+\frac{a d}{2}+d\right) \lambda & \\ +\left(c+\frac{a d}{2}\right)(d+1)\left(\frac{1}{2}-\frac{1}{a}\right)(c+1-c \lambda) & \text { if } 1+\frac{1}{c+a d} \leq \lambda<1+\frac{1}{c} \\ d(d+1)-d\left(c+\frac{a d}{2}+d\right) \lambda & \text { if } 1+\frac{1}{c} \leq \lambda<1+\frac{1}{d},\end{cases}
$$

and for $c \leq d$

$$
g_{R, \mathbf{m}}(\lambda)= \begin{cases}\left(c+\frac{a d}{2}+d\right) \lambda & \text { if } 0 \leq \lambda<1 \\ -\left(c+\frac{a d}{2}+d\right)\left(c d+\frac{a d^{2}}{2}+c+\frac{a d}{2}+d\right) \lambda & \\ +\left(c+\frac{a d}{2}+d\right)(d+1)\left(c+\frac{a d}{2}+1\right) & \text { if } 1 \leq \lambda<1+\frac{1}{c+a d} \\ d(d+1)-d\left(c+\frac{a d}{2}+d\right) \lambda & \\ +\left(c+\frac{a d}{2}\right)(d+1)\left(\frac{1}{2}-\frac{1}{a}\right)(c+1-c \lambda) & \text { if } 1+\frac{1}{c+a d} \leq \lambda<1+\frac{1}{d} \\ \left(c+\frac{a d}{2}\right)(d+1)\left(\frac{1}{2}-\frac{1}{a}\right)(c+1-c \lambda) & \\ +d\left(c+\frac{a d}{2}+\frac{a}{2}\right)\left(c+\frac{a d}{2}+d\right)(\lambda-1) & \\ -d\left(2+\frac{a}{2}\right)\left(c+\frac{a d}{2}+\frac{a}{2}\right) & \text { if } 1+\frac{1}{d} \leq \lambda<1+\frac{a+1}{a d+c} \\ c\left(\frac{1}{2}-\frac{1}{a}\right)(c+1-c \lambda) & \text { if } 1+\frac{a+1}{a d+c} \leq \lambda<\frac{1}{c} .\end{cases}
$$




\section{Appendix A}

We recall the following notion of relative volume for a convex polytope, given by $\mathrm{R}$. Stanley ([16, see page 238]).

Definition 47 Let $Q \subset \mathbb{R}^{d}$ be an integral convex polytope of dimension $m$. Let $A(Q)$ be the affine span of $Q$. Then $A(Q) \cap \mathbb{Z}^{d}$ is an abelian group of rank $m$. Choose an invertible affine transformation $\varphi: A(Q) \longrightarrow \mathbb{R}^{m}$ such that $\varphi\left(A(Q) \cap \mathbb{Z}^{d}\right)=\mathbb{Z}^{m}$. Then the image $\varphi(Q)$ of $Q$ is an integral polytope and relative volume of $Q$ is defined to be volume of $\varphi(Q)$.

If $Q$ is an $m$-dimensional rational polytope, then there is $n>0$ such that $n Q$ is an integral polytope. We define $\operatorname{rVol}_{m}(Q):=\operatorname{rVol}_{m}(n Q) / n^{m}$ and $\operatorname{rVol}_{m_{1}}(Q):=0$ if $m_{1}>m$.

Moreover, if $Q=\cup_{i} Q_{i}$, is a finite union of $d^{\prime}$-dimensional convex rational polytopes $Q_{i}$ such that $\operatorname{dim}\left(Q_{i} \cap Q_{j}\right)<d^{\prime}$, for $Q_{i} \neq Q_{j}$, then we define $\mathrm{rVol}_{d^{\prime}} Q=\sum_{i} \mathrm{rVol}_{d^{\prime}} Q_{i}$ and $\operatorname{rVol}_{d^{\prime \prime}} Q=0$, if $d^{\prime \prime}>d^{\prime}$. One can show that $\operatorname{rVol}_{d^{\prime}} Q$ is independent of the choice of the finite decomposition $Q=\cup_{i} Q_{i}$.

Lemma 48 Let $F$ be a rational convex polytope of dimensiond -1 in $\mathbb{R}^{d}$ satisfying the following: $A(F) \cap\{z=i\} \cap \mathbb{Z}^{d} \neq \phi$, for every $i \in \mathbb{Z}$, where $A(F)$ denotes the affine hull of the polytope $F$, and $z$ is the coordinate function on $\mathbb{R}^{d}$. Let $\varphi: A(F) \longrightarrow \mathbb{R}^{d-1}$ be an invertible affine transformation such that $\varphi\left(A(F) \cap \mathbb{Z}^{d}\right)=\mathbb{Z}^{d-1}$.

Then there is $z_{1} \in A(F) \cap\{z=1\} \cap \mathbb{Z}^{d}$ and $\left\{x_{1}, \ldots, x_{d-2}\right\} \in A(F) \cap\{z=0\} \cap \mathbb{Z}^{d}$ such that $\left\{\varphi\left(x_{1}\right), \ldots, \varphi\left(x_{d-2}\right), \varphi\left(z_{1}\right)\right\}$ is a basis of $\mathbb{R}^{d-1}$ and

$$
\mathrm{rVol}_{d-2}(F \cap\{z=i\})=\operatorname{Vol}_{d-2}\left(\varphi(F) \cap \pi_{\varphi}^{-1}\{i\}\right),
$$

where $\pi_{\varphi}: \mathbb{R}^{d-1}=\varphi(A(F)) \longrightarrow \mathbb{R}$ is the map given by $\sum \lambda_{i} \varphi\left(x_{i}\right)+\lambda_{d-1} \varphi\left(z_{1}\right) \rightarrow$ $\lambda_{d-1}$.

Proof Reduction By hypothesis, we can choose $x_{0} \in A(F) \cap \mathbb{Z}^{d}$. Let $y_{0}=\varphi\left(x_{0}\right)$. Let $\psi_{x_{0}}: \mathbb{R}^{d} \longrightarrow \mathbb{R}^{d}$ and $\psi_{-y_{0}}^{\prime}: \mathbb{R}^{d-1} \longrightarrow \mathbb{R}^{d-1}$ denote the translation maps given by the elements $x_{0}$ and $-y_{0}$, respectively. Then replacing $\varphi$ by $\psi_{-y_{0}}^{\prime} \circ \varphi \circ \psi_{x_{0}}$ : $A(F)-x_{0} \longrightarrow \mathbb{R}^{d-1}$ and $A(F)$ by $A(F)-x_{0}$, we can assume that $\varphi$ is a linear transformation and $A(F)$ is a $\mathbb{R}$-vector space. Let $V_{n}=A(F) \cap\{z=n\}$, and then $V_{0}$ is a $d-1$-dimensional vector subspace. We choose $z_{1} \in V_{1}$.

Claim (1) $V_{n}=V_{0}+n z_{1}$ and (2) $\varphi\left(V_{n}\right)=\varphi(A(F)) \cap \pi_{\varphi}^{-1}\{n\}$. There exists a basis $\left\{\varphi\left(x_{1}\right), \ldots, \varphi\left(x_{d-2}\right), \varphi\left(z_{1}\right)\right\} \in \mathbb{Z}^{d-1}$ of $\varphi(A(F))$.

Proof of the Claim (1) Let $x_{1}, \ldots, x_{d-2}$ be a set of generators of the free abelian group $V_{0} \cap \mathbb{Z}^{d}$. Then $\left\{x_{1}, \ldots, x_{d-2}\right\}$ is a vector space basis for $V_{0}$. Therefore $\left\{\varphi\left(x_{1}\right), \ldots, \varphi\left(x_{d-2}\right), \varphi\left(z_{1}\right)\right\}$ is a basis for $\varphi(A(F))=\mathbb{R}^{d-1}$. Hence $\varphi(A(F))=$ $\sum_{i} \mathbb{R} \varphi\left(x_{i}\right)+\mathbb{R} \varphi\left(z_{1}\right)$. It is obvious that $V_{0}+n z_{1} \subseteq V_{n}$. Let $y \in V_{n}$, and then $y=\sum_{i} \lambda_{i} x_{i}+\lambda_{0} z_{1}$. Comparing the $(d-1)^{t h}$ coordinate we get $\lambda_{0}=n$ and therefore $y \in V_{0}+n z_{1}$. (b) Let $y \in V_{n}=V_{0}+n z_{1}$. Then $y=\left(\sum_{i} \lambda_{i} x_{i}\right)+n z_{1}$. This implies $\varphi\left(V_{n}\right)=\sum_{i} \mathbb{R} \varphi\left(x_{i}\right)+\mathbb{R} \varphi\left(z_{1}\right) \subseteq \varphi(A(F)) \cap \pi_{\varphi}^{-1}\{n\}$. This proves the claim. 
Since $\varphi\left(V_{n}\right)=\left(\sum_{i} \mathbb{R} \varphi\left(x_{i}\right)\right)+n \varphi\left(z_{1}\right)$ and $V_{n} \cap \mathbb{Z}^{d}=\sum_{i=1}^{d-2} \mathbb{Z} x_{i}+\varphi\left(z_{1}\right)$, we have $\varphi\left(V_{n} \cap \mathbb{Z}^{d}\right) \subseteq \varphi\left(V_{n}\right) \cap \mathbb{Z}^{d-1}$. Now

$$
\varphi\left(A(F) \cap \mathbb{Z}^{d}\right)=\cup_{n} \varphi\left(V_{n} \cap \mathbb{Z}^{d}\right) \subseteq \cup_{n}\left(\varphi\left(V_{n}\right) \cap \mathbb{Z}^{d-1}\right) \subseteq \varphi\left(A(F) \cap \mathbb{Z}^{d-1}\right),
$$

This implies $\varphi\left(V_{n} \cap \mathbb{Z}^{d}\right)=\varphi\left(V_{n}\right) \cap \mathbb{Z}^{d-1}$, for all $n \in \mathbb{Z}$. Hence $\operatorname{rVol}_{d-2}\left(V_{n}\right)=$ $\operatorname{Vol}_{d-2} \varphi\left(V_{n}\right)=\operatorname{Vol}_{d-2}\left[\varphi(A(F)) \cap \pi_{\varphi}^{-1}\{n\}\right]$. This proves the lemma.

Lemma 49 If $Q$ is a convex rational polytope in $\mathbb{R}^{d}$ and $Q_{\lambda}=Q \cap\{z=\lambda\}$, for $\lambda \in \mathbb{R}$, then there is a constant $C_{Q}$ (independent of $\lambda$ ) such that $i\left(Q_{\lambda}, n\right)=c_{\lambda}(n) n^{\text {dim }} Q_{\lambda}$ with $\left|c_{\lambda}(n)\right| \leq C_{Q}$.

Proof For $\lambda \in \mathbb{R}$, let $d_{\lambda}=\operatorname{dim}\left(Q_{\lambda}\right)$. Let $P_{\lambda}$ be an integral $d_{\lambda}$-dimensional cube with length of each side $=c_{\lambda}$ and $Q_{\lambda} \subseteq P_{\lambda}$. Then $i\left(Q_{\lambda}, n\right) \leq i\left(P_{\lambda}, n\right) \leq c_{\lambda}^{d_{\lambda}} n^{d_{\lambda}}$. Since $Q$ is a bounded set, there exists a constant $C_{Q}^{\prime}$ such that, for any $\lambda$ we can choose $P_{\lambda}$ with $\left|c_{\lambda}\right| \leq C_{Q}^{\prime}$. Hence the lemma follows by taking $C_{Q}=\left(C_{Q}^{\prime}\right)^{\operatorname{dim}(Q)}$.

\section{References}

1. Bruns, W., Gubeladze, J.: Divisorial linear algebra of normal semigroup rings. Algebr. Represent. Theory 6(2), 139-168 (2003)

2. Chan, C.Y., Kurano, K.: Hilbert-Kunz functions over rings regular in codimension one. Comm. Algebra 44(1), 141-163 (2016)

3. Cox, D.A., Little, J.B., Schenck, H.K.: Toric Varieties, Graduate Studies in Mathematics. American Mathematical Society, Providence (2011)

4. Ehrhart, E.: Sur les polyèdres rationnels homothétiques à n dimensions. C. R. Acad. Sci. Paris Ser. A 254, 616-618 (1962)

5. Eto, K.: Multiplicity and Hilbert-Kunz multiplicity of monoid rings. Tokyo J. Math. 25(2), 241-245 (2002)

6. Fulton, W.: Introduction to Toric Varieties. Annals of Mathematics Studies, The William H. Roever Lectures in Geometry, vol. 131. Princeton University Press, Princeton (1993)

7. Henk, M., Linke, E.: Note on the coefficients of rational Ehrhart quasi-polynomials of Minkowskisums. Online J. Anal. Comb. 10, 12 pp (2015)

8. Hochster, M., Yao, Y.: Second coefficients of Hilbert-Kunz functions for domains. Preliminary preprint available at http://www.math.lsa.umich.edu/ hochster/hk.pdf

9. Huneke, C., McDermott, M.A., Monsky, P.: Hilbert-Kunz functions for normal rings. Math. Res. Lett. 11, 539-546 (2004)

10. Kurano, K.: The singular Riemann-Roch theorem and Hilbert-Kunz functions. J. Algebra 304, 487499 (2006)

11. Linke, E.: Rational Ehrhart quasi-polynomials. J. Combin. Theory Ser. A 118(7), 1966-1978 (2011)

12. McMullen, P.: Lattice invariant valuations on rational polytopes. Arch. Math. 31, 509-516 (1978/1979)

13. Mondal, M., Trivedi, V.: Hilbert-Kunz density function and asymptotic Hilbert-Kunz multiplicity for projective toric varieties. J. Algebra 520, 479-516 (2019)

14. Monsky, P.: The Hilbert-Kunz function. Math. Ann. 263(1), 43-49 (1983)

15. Schneider, R.: Convex Bodies: The Brunn-Minkowski Theory, 2nd edn. Cambridge University Press, Cambridge (2013)

16. Stanley, R.P.: Enumerative Combinatorics, vol. 1. Cambridge University Press, Cambridge (1997)

17. Trivedi, V.: Hilbert-Kunz functions of a Hirzebruch surface. J. Algebra 457, 405-430 (2016)

18. Trivedi, V.: Hilbert-Kunz density function and Hilbert-Kunz multiplicity. Trans. Amer. Math. Soc. 370(12), 8403-8428 (2018)

19. Trivedi, V.: Asymptotic Hilbert-Kunz multiplicity. J. Algebra 492, 498-523 (2017) 
20. Trivedi, V.: Towards Hilbert-Kunz multiplicity in characteristic 0. Nagoya Math. J., 1-43 (2018). https://doi.org/10.1017/nmj.2018.7

Publisher's Note Springer Nature remains neutral with regard to jurisdictional claims in published maps and institutional affiliations. 